\title{
Troponin structure and function: a view of recent progress
}

\author{
Steven Marston ${ }^{1}\left[\right.$ ] Juan Eiros Zamora ${ }^{1}$
}

Received: 9 January 2019 / Accepted: 12 April 2019 / Published online: 27 April 2019

(c) The Author(s) 2019

\begin{abstract}
The molecular mechanism by which $\mathrm{Ca}^{2+}$ binding and phosphorylation regulate muscle contraction through Troponin is not yet fully understood. Revealing the differences between the relaxed and active structure of cTn, as well as the conformational changes that follow phosphorylation has remained a challenge for structural biologists over the years. Here we review the current understanding of how $\mathrm{Ca}^{2+}$, phosphorylation and disease-causing mutations affect the structure and dynamics of troponin to regulate the thin filament based on electron microscopy, X-ray diffraction, NMR and molecular dynamics methodologies.
\end{abstract}

Keywords Troponin $\cdot$ Thin filaments $\cdot \mathrm{Ca}^{2+} \cdot$ Phosphorylation $\cdot$ Muscle regulation $\cdot$ Mutation

\section{Introduction}

The molecular mechanism by which $\mathrm{Ca}^{2+}$ binding and phosphorylation regulate muscle contraction through Troponin is not yet fully understood. Revealing the differences between the relaxed and active structure of cTn, as well as the conformational changes that follow phosphorylation has remained a challenge for structural biologists over the years (Fig. 1). Here we review the current understanding of how $\mathrm{Ca}^{2+}$, phosphorylation and mutations affect the structure and dynamics of troponin to regulate the thin filament.

The most important achievement in troponin structure was the publication of a partial crystal structure of the core domain of human cardiac troponin (cTn) in the $\mathrm{Ca}^{2+}$ saturated state by Takeda et al. (2003) (Fig. 2). This structure reveals that in this state cTn adopts an L-shaped conformation, with different domains connected by flexible linkers. A rigid coiled-coil domain is observed between TnT and TnI and is referred to as the 'IT arm' in the literature. Additionally, the interaction between the $\mathrm{N}$-terminal domain of cTnC and the C-terminal regions of cTnI ('switch peptide') is apparent and is usually referred to as the 'regulatory head'. A later publication reported the activated state of chicken

Steven Marston

s.marston@imperial.ac.uk

Juan Eiros Zamora

j.eiros-zamora14@imperial.ac.uk

1 NHLI and Chemistry Departments, Imperial College

London, W12 0NN London, UK fast skeletal muscle troponin (sTn) in high resolution and a low-resolution structure of the inactive state (Vinogradova et al. 2005). These structures highlight the differences between the sTn and cTn isoforms. The cTnI isoform has an extended $\mathrm{N}$-terminal region of 30 amino acids which is not present in the sTn isoform. This region was not present in the crystal structure of Takeda (2003) due to its disordered nature. Although the overall arrangement of the subunits of cardiac and skeletal muscle troponin is similar, the location of the regulatory head is different, with a smaller angle being formed between it and the IT arm in the sTn isoform. Additionally, in the $\mathrm{Ca}^{2+}$ form,the linker that joins the two domains of $\mathrm{TnC}$ is helical in the sTn molecule, while in cTn it is disordered. Finally, the $\mathrm{Ca}^{2+}$-free structure of sTn indicates dissociation of the switch peptide of $\mathrm{cTnI}$ from the hydrophobic cleft that is bound to in the $\mathrm{Ca}^{2+}$-saturated conformation.

The cTn crystal structure presents the orientation between the IT arm $(\alpha$-helical coiled-coil structure formed from part of cTnI and cTnT) and the regulatory head (both terminal regions of $\mathrm{cTnI}$ and the $\mathrm{N}$-terminal domain of troponin $\mathrm{C}$ (NcTnC). Further nuclear magnetic resonance (NMR) studies focused on revealing the sections of the complex that are missing from Takeda's structure, such as the cardiac-specific NcTnI region, the cTnI inhibitory region, the C-terminal regions of both cTnI and $\mathrm{cTnT}$ and all of the $\mathrm{N}$ terminal domain of TnT (over 200 residues). An NMR structure of the $\mathrm{N}$ terminus of troponin I ( $\mathrm{NcTnI}$ ) region published by Howarth (2007), both in the unphosphorylated and bisphosphorylated forms, showed substantial conformational 
Fig. 1 Model of the muscle thin filament based on the coordinates published by Pirani et al. (2006) showing the likely arrangement of actin (white), tropomyosin (red) and troponin (TnC pink, TnI magenta, TnT yellow), based on electron microscopy and X-ray diffraction. The barbed end ( $\mathrm{Z}$ - band end) of the actin filament is on the left of the figure. (Color figure online)

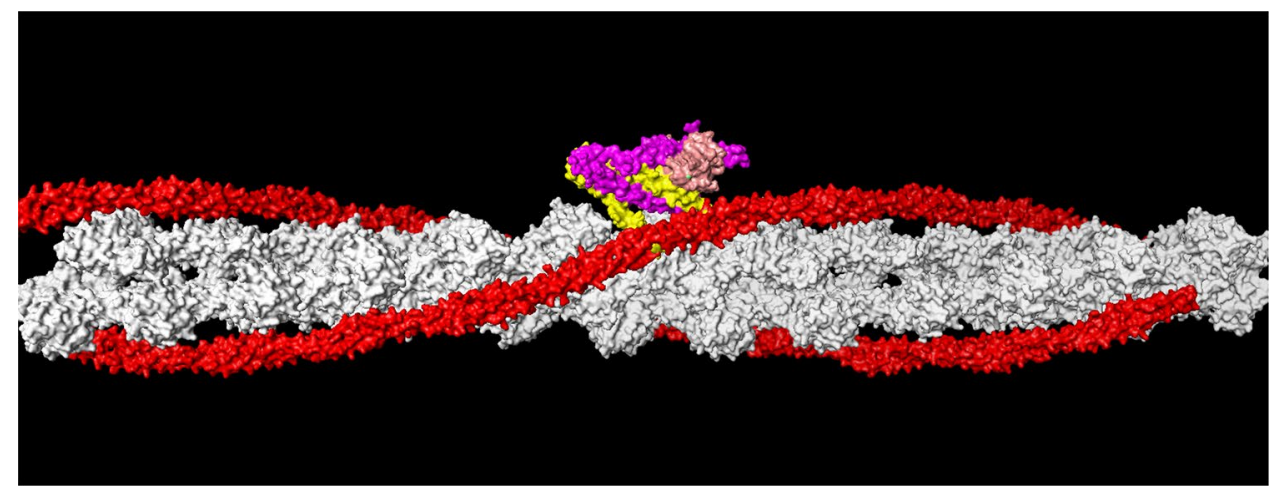

Fig. 2 Front and back view of the $46 \mathrm{kDa}$ core domain of human cardiac Troponin in the $\mathrm{Ca}^{2+}$-activated form. $\mathrm{TnC}$ is depicted in blue, TnT in green and $\mathrm{TnI}$ in red. PDB accession code 1J1D (Takeda et al. 2003). (Color figure online)
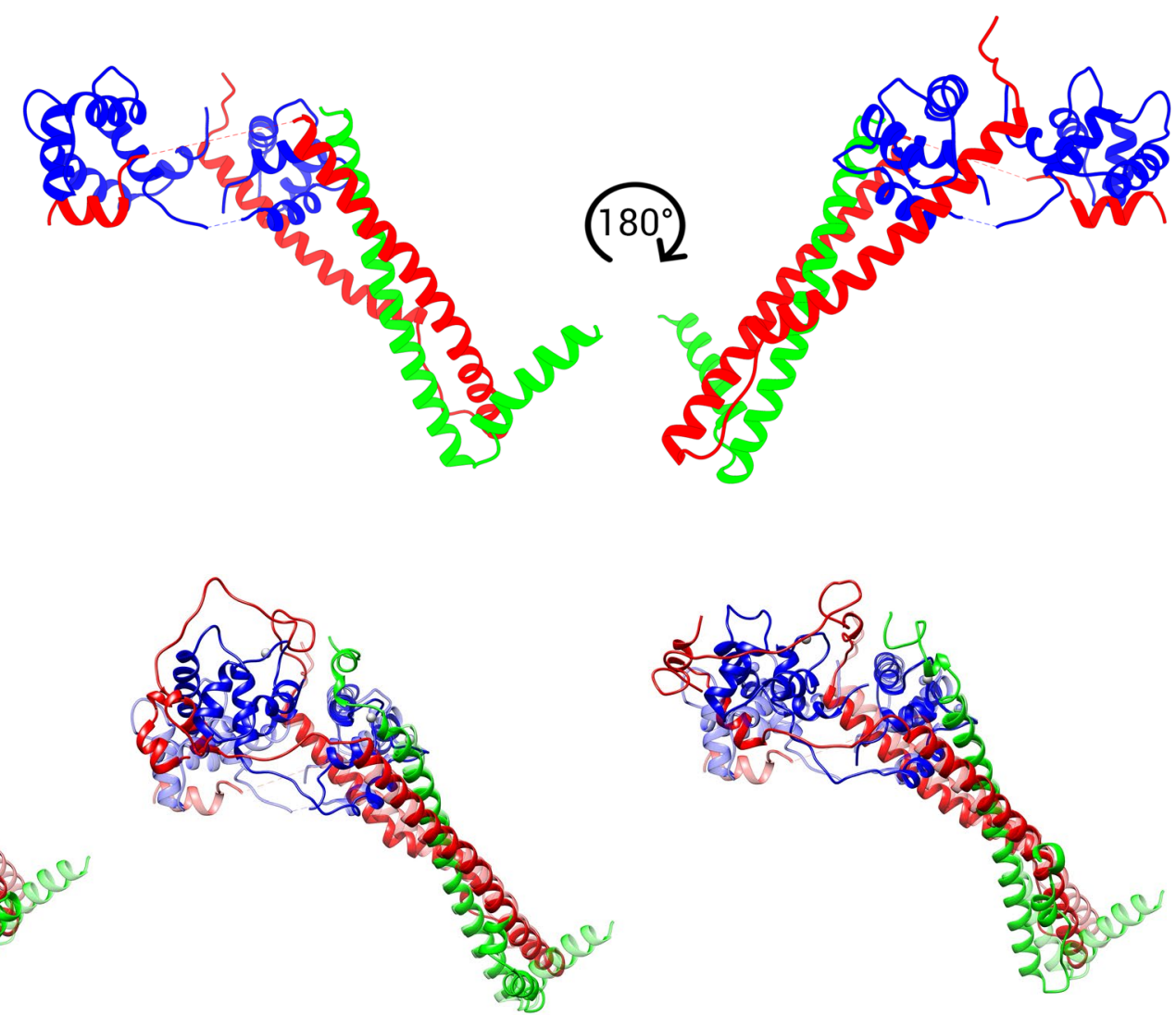

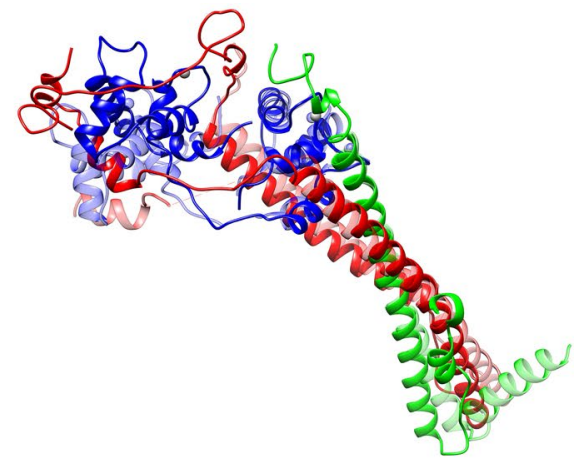

Fig. 3 The three most populated structures of human cardiac troponin determined by molecular dynamics simulations overlaid over the Takeda et al. structure of hcTn (pale structure) TnC is depicted in blue, TnT in green and TnI in red (Zamora 2019). (Color figure online)

differences between the two. Recent NMR studies have described this peptide as an intrinsically disordered region (IDR) in the non-phosphorylated form. Its interaction with $\mathrm{NcTnC}$ also seemed to position it relative to the IT arm, and possibly modulate its interaction with the cTnI switch peptide. There are conformational changes after phosphorylation of NcTnI that appear to disrupt this interaction (Hwang et al. 2014).

Recently the structure of the cardiac troponin core has been investigated by molecular dynamics simulations
(Cheng et al. 2014; Papadaki and Marston 2016; Zamora et al. 2016); (Fig. 3).

These studies confirm the structure of troponin derived from X-ray crystallography for the rigid parts of the molecule and provide information about the structure and dynamics of the intrinsically disordered segments. The regulatory head and the IT arm are found to be relatively rigid and similar to the crystal structure but the relative disposition of the two domains is extremely variable. The structure of troponin cannot be realistically represented by any static model, 
but, for comparison with the X-ray model, we show the most populated structures of the three most probable macrostates of cardiac troponin with $\mathrm{Ca}^{2+}$ bound derived from MD calculations (Zamora 2019; Zamora et al. 2016) (Fig. 3).

The location of NcTnI near NcTnC, the hinge motion of the linker peptide region between the regulatory head and the $\mathrm{C}$ terminus/IT arm and the potential interactions of the C-terminus of troponin $\mathrm{T}$ with the $\mathrm{TnI}$ and $\mathrm{TnC}$ in the hinge region is evident from these structures.

\section{Troponin C}

The troponin $\mathrm{C}(\mathrm{TnC})$ molecule owes its name to the fact that it is the $\mathrm{Ca}^{2+}$ sensing subunit of the Tn complex. In the human heart, it is expressed as a 161-residue long protein made up of two globular domains joined by a flexible hydrophobic linker. It has a molecular weight of around $18 \mathrm{kDa}$. TnC contains nine short $\alpha$ helices named in alphabetical order except for the first, helix N (Figs. 4 and 5). In total, TnC contains four structural motifs known as EF-hands capable of binding positive divalent cations. Each EF-hand consists of two $\alpha$-helices interconnected by a loop with negatively charged residues that coordinate the positive ion. EF hand I (residues 16 to 51) and EFhand II (residues 52 to 87 ) have a low affinity for $\mathrm{Ca}^{2+}$ $\left(K_{a} \approx 10^{5} \mathrm{M}^{-1}\right)$ but are highly selective to it. These different behaviours have resulted in the naming of the $\mathrm{Ca}^{2+}$ ions that bind to site I and II as the 'catalytic' or 'regulatory' $\mathrm{Ca}^{2+}$, while sites III (residues 92 to 127) and IV (residues 128 to 161) are known as'structural' $\mathrm{Ca} / \mathrm{Mg}$ sites; these are always occupied under physiological conditions
Fig. 4 The cTnC molecule with its nine helices highlighted. Subunits cTnI and cTnT are transparent for clarity. The troponin structure is the original Takeda structure with the disordered segments of TnI and TnT modelled (Zamora et al. 2016)
Fig. 5 Sequence and secondary structure of cTnC N-terminal domain. Numbering is for human cardiac troponin $\mathrm{C}$ (P63316)

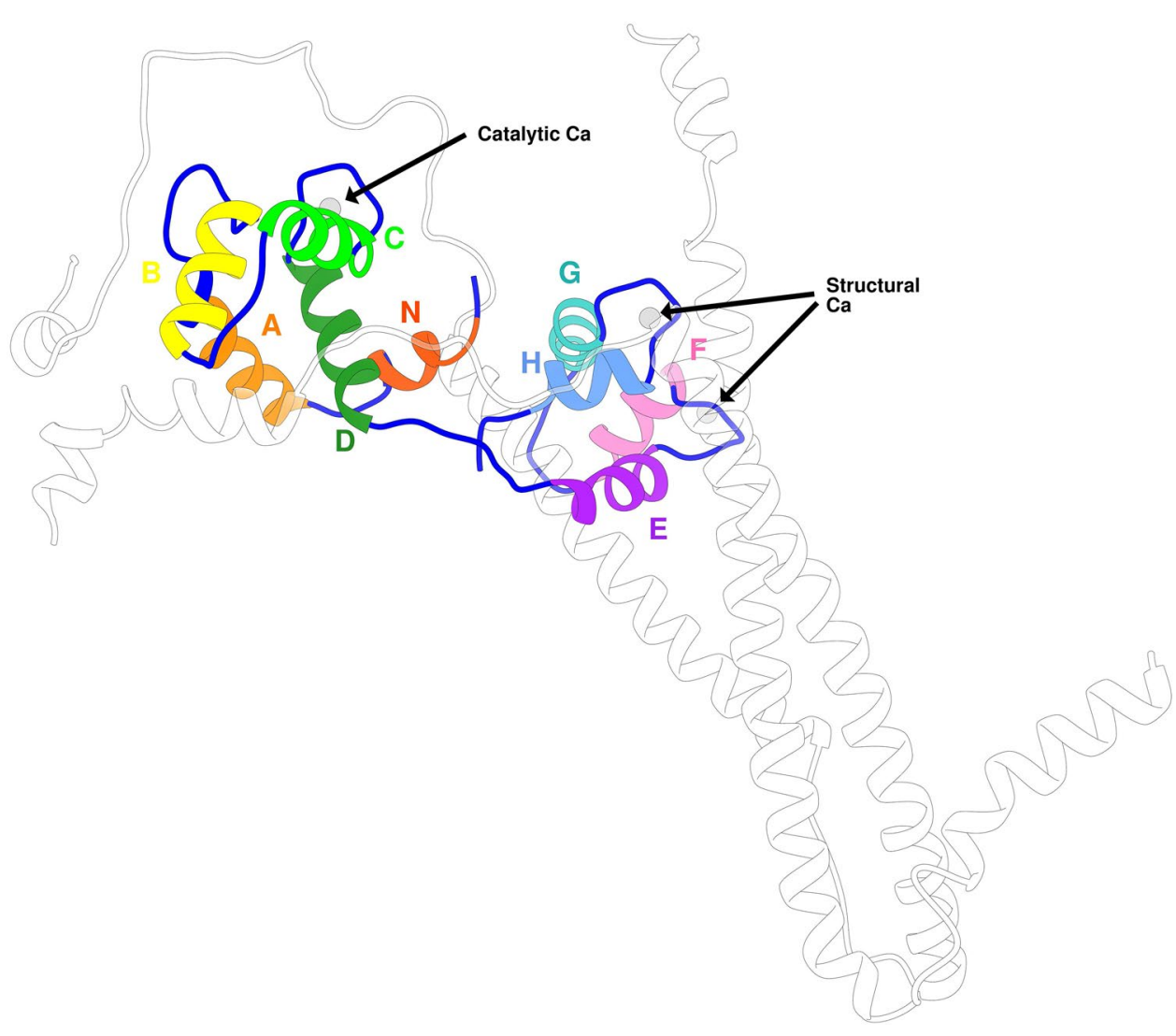

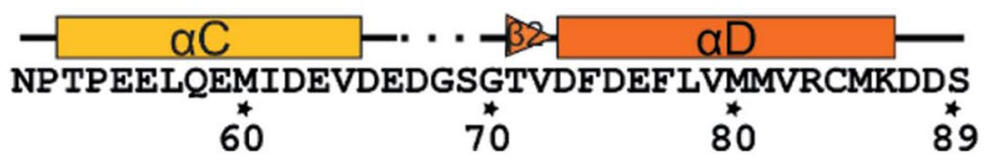


Table 1 Standard nomenclature and numbering for the structural elements of human cardiac TnC (P63316)

\begin{tabular}{cc}
\hline Residues & Region \\
\hline $3-9$ & Helix N \\
$14-25$ & Helix A \\
$38-47$ & Helix B \\
$54-64$ & Helix C \\
$74-85$ & Helix D \\
$94-104$ & Helix E \\
$114-123$ & Helix F \\
$130-140$ & Helix G \\
$150-157$ & Helix H \\
\hline $16-51$ & EF hand I \\
$52-87$ & EF hand II \\
$92-127$ & EF hand III \\
$128-161$ & EF hand IV \\
\hline $1-87$ & N terminal globular domain \\
$88-91$ & Flexible linker \\
$92-161$ & C terminal globular domain \\
\hline
\end{tabular}

$\left(K a \approx 10^{7} \mathrm{M}^{-1}\right.$ ), and other divalent cations such as $\mathrm{Mg}^{2+}$ and $\mathrm{Cd}^{2+}$ can also bind to these sites. In the cardiac isoform, the first EF-hand does not bind $\mathrm{Ca}^{2+}$ at any concentration thus site II is alone responsible for $\mathrm{Ca}^{2+}$ sensing during muscle contraction (Figs. 4, 5, Table 1).

\section{N-terminal domain of TnC}

In its role as $\mathrm{Ca}^{2+}$ sensor, troponin $\mathrm{C}$ undergoes an extensive concerted structural rearrangement when $\mathrm{Ca}^{2+}$ binds from the so-called 'closed' state to an 'open' state. The two EF hands move closer to each other with the formation of a short hydrogen-bonded beta-sheet motif between them and reorientation of the helices resulting in the opening of a hydrophobic patch that creates a binding site for the switch peptide of troponin I (Figs. 5, 6).

\section{Troponin I}

Troponin I (TnI) is expressed in three different isoforms: cardiac, slow skeletal and fast skeletal muscle TnI. During embryonic development, slow skeletal $\mathrm{TnI}$ is expressed in the cardiac tissue, but it is replaced by cardiac TnI (cTnI) after birth (Marston and Redwood 2003), with the expression of this isoform being exclusive to the cardiac tissue. The mature form of human cTnI consists of a 210-residue long protein (209 after the first Met residue is removed) with a molecular mass of approximately $24 \mathrm{kDa}$. This protein is arranged as four $\alpha$ helices intercalated by flexible disordered regions. Structurally, it can be classified into the following domains: the cardiac-exclusive $\mathrm{N}$ terminal domain (NcTnI), the structurally rigid IT arm, the "inhibitory" peptide, the switch peptide and the C-terminal domain (Fig. 7 and Table 2).

The NcTnI region is exclusively expressed in the cardiac isoform of TnI. It consists of an acidic region (residues 2 to 11) and a Xaa-Pro motif (residues 12 to 18) that can form a short and unstable $\alpha$ helix. In this region two adjacent Serine residues are located (Ser 22 and Ser 23) which are the targets of PKA-mediated phosphorylation. A structure has been proposed for this peptide in isolation, however in the whole troponin molecule it is disordered, although its location over the $\mathrm{N}$-terminal domain of $\mathrm{TnC}$ has been predicted from modelling and molecular dynamics simulations (Cheng et al. 2014; Howarth et al. 2007; Hwang et al. 2014; Zamora et al. 2016) (Figs. 3, 7).

The IT arm is a rigid coiled-coil of $\alpha$ helices of cTnI and cTnT. It is formed by the directionally-opposed $\alpha$ helices $\mathrm{H} 1$ and $\mathrm{H} 2$ (residues 43 to 79 and 90 to 135, respectively). These two helices are connected by a flexible U-turn (residues 80 to 89). The function of the IT arm is structural, as it is the least mobile region of the Tn complex. It serves as an anchoring region for the C-terminal domain of troponin $\mathrm{C}$.

Upstream of helix $\mathrm{H} 2$ is the so-called 'inhibitory peptide' of cTnI (residues 137 to 148), which contains six positively charged residues. This short region owes its name to the observation that the peptide by itself can interact with actin-tropomyosin and inhibit actomyosin ATPase at a substoichiometric ratio to actin (Van Eyk et al. 1997). The name is, however, misleading: when incorporated into the whole troponin it is a flexible linker between the TnC $\mathrm{N}$ terminal domain and the TnC C-terminus/IT arm domain and is not directly involved in inhibition. The existing structural studies on this region have provided conflicting views on whether this region has any defined structure or not.

The switch peptide of cTnI includes helix $\mathrm{H} 3$ (residues 151 to 162) which at high $\mathrm{Ca}^{2+}$ concentration interacts with the $\mathrm{N}$ terminal region of $\mathrm{cTnC}$ through the hydrophobic patch that is otherwise closed (Fig. 6b). The binding of $\mathrm{TnI}$ to $\mathrm{TnC}-\mathrm{Ca}^{2+}$ is the key interaction of muscle 

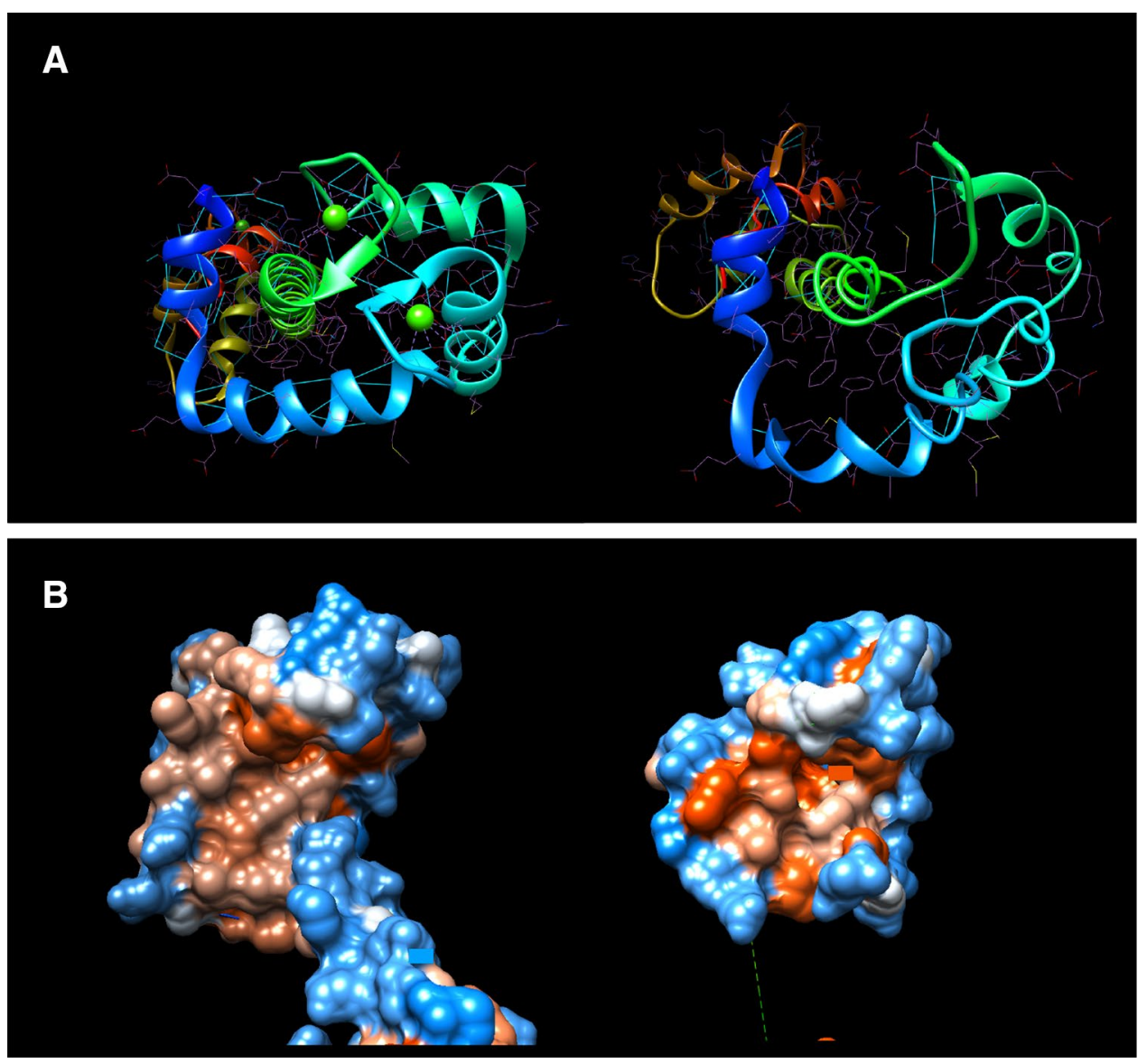

Fig. $6 \mathrm{Ca}^{2+}$ dependent changes in the structure of the N-terminal lobe of skeletal troponin C. These images are from the X-ray structure of whole skeletal muscle troponin (1ytz and 1yv0) (Vinogradova et al. 2005) with TnI and TnT edited out for clarity. Thin blue lines are putative hydrogen bonds. Thin red lines are the residues of the hydrophobic patch. a Ribbon diagram of (left) the $\mathrm{Ca}^{2+}$-saturated sTnC and (right) $\mathrm{Ca}^{2+}$-free $\mathrm{TnC}$, viewed from above the short beta sheet formed by amino acids just preceding helices $\mathrm{B}$ and $\mathrm{D}$. This structure is dis- rupted, the two $\mathrm{EF}$ hands $(\mathrm{AB}$ and $\mathrm{CD})$ move away from each other and the angle between helix $\mathrm{A}$ and $\mathrm{B}$ changes from $135^{\circ}$ to $81^{\circ}$ in the absence of $\mathrm{Ca}^{2+}$. b Hydrophobicity surface rendering of sTnC, viewed from the underneath. Maximum hydrophobicity is brown and minimum is blue. In the $\mathrm{Ca}^{2+}$ bound state an extensive hydrophobic surface is presented to the TnI switch peptide (see Fig. 8) (left) that is closed off in the absence of $\mathrm{Ca}^{2+}$ (right). (Color figure online)

none of the models is favoured, instead, $\mathrm{TnI} \mathrm{C}$ terminus is viewed as intrinsically disordered and can exist in many low energy conformers that are kinetically connected through an ensemble of disordered transition states. In general, the conformers are extended and it has been suggested that $\mathrm{TnI}$ C-terminal inhibitory binding to actin could be described by a "fly-casting mechanism" that would allow $\mathrm{TnI} C$ terminus to explore the surface of actin at a distance of 5-7 $\mathrm{nm}$ from the troponin core. Upon finding its contact site it would form a folded complex on actin, thus "reeling in" the whole troponin complex to the position that it occupies once $\mathrm{TnI}$ is bound to actin (Blumenschein et al. 2006; Shoemaker et al. 2000). 
Fig. 7 The cTnI molecule with its relevant structural regions highlighted. Subunits cTnC and cTnT are transparent for clarity. The switch peptide corresponds to Helix H3. Residues 172 to 210 of cTnI are not present in this structure (Zamora et al. 2016)

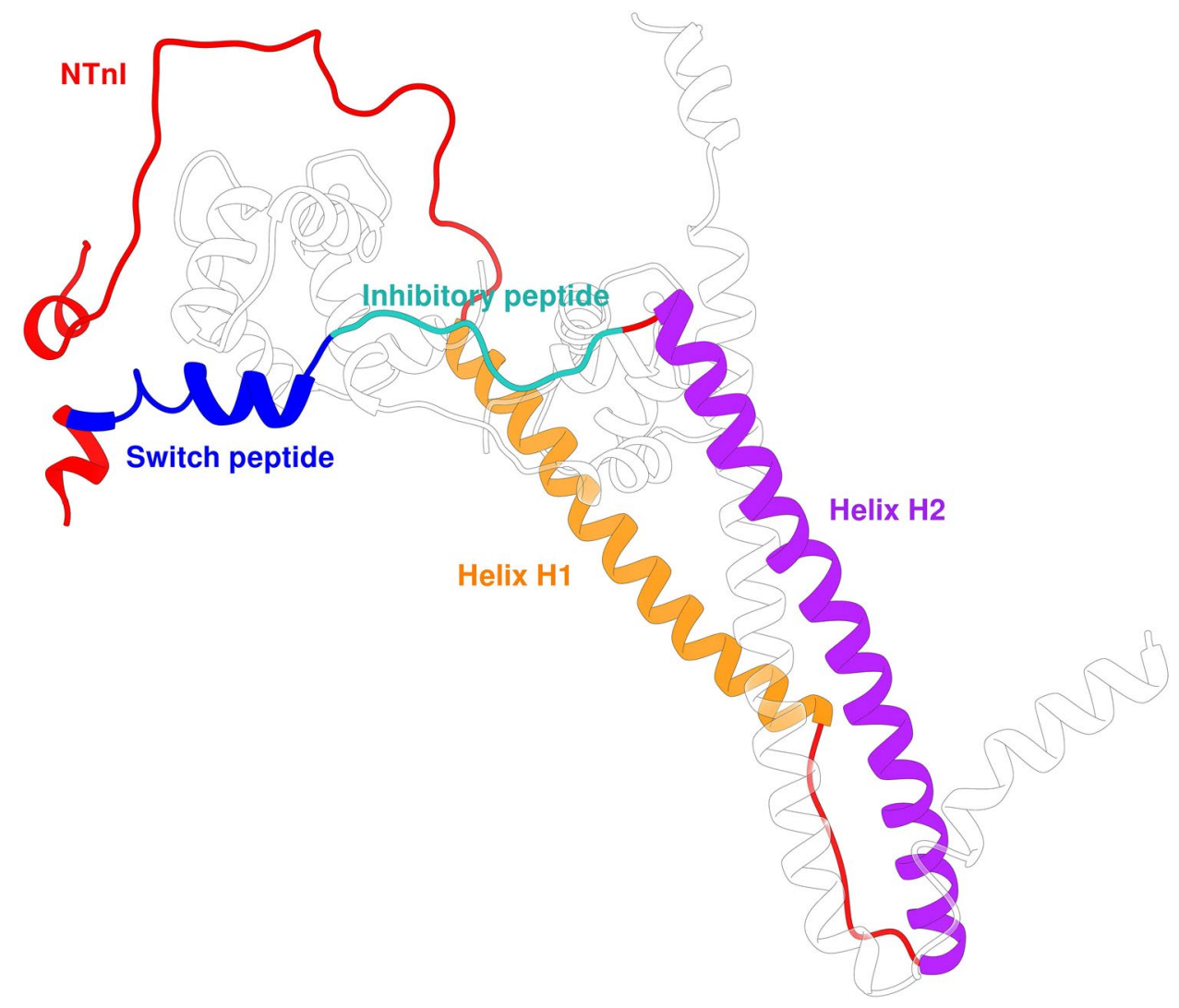

Table 2 Standard nomenclature and sequence numbering for the different structural regions of human cardiac troponin I (P19429)

\begin{tabular}{cc}
\hline Residues & Region \\
\hline $43-79$ & Helix H1 \\
$80-89$ & Flexible U-turn \\
$90-135$ & Helix H2 \\
$151-162$ & Helix H3 \\
$163-188$ & Helix H4 \\
\hline $2-32$ & N terminal domain \\
$42-136$ & IT arm \\
$137-148$ & Inhibitory peptide \\
$149-164$ & Switch peptide \\
$163-210$ & C terminal domain \\
\hline
\end{tabular}

\section{Troponin T}

The troponin $\mathrm{T}(\mathrm{TnT})$ subunit is the largest of the three and mainly has a structural role. It is responsible for the fixation of the complex to the thin filament surface and the positioning of the core domain of cTn relative to the direction of the actin filament. Similarly to cTnI, there exist several isoforms of this protein which are differentially expressed in the heart, the fast and slow skeletal muscle, as well as different isoforms expressed during embryonic development. Each tissue-specific gene can express a variety of protein isoforms by alternative splicing. The description here is for the dominant adult cardiac muscle TnT isoform (TNNT2 gene isoform 6 (P45379-6), commonly referred to as T3 (Anderson et al. 1991; Bayliss et al. 2012).

The mature form in the adult human heart is a 288-residue long protein (287 after the first Met residue is removed) with an approximate molecular weight of $36 \mathrm{kDa}$. Despite its structural role, the majority of the cTnT molecule has not been resolved experimentally, and most of what is known about has been gathered from studies of partial structures.

The Troponin $\mathrm{T}$ amino acid sequence is divided into three structural domains: The $\mathrm{N}$ terminal domain, the linking domain and the $\mathrm{C}$ terminal domain (Table 3). The $\mathrm{C}$ terminal domain is part of the core troponin complex whilst the rest is associated with tropomyosin. The $\mathrm{N}$-terminal segment, named TnT1, has an extended structure that includes regions of single alpha-helix that bind to tropomyosin. The X-ray diffraction study of (Cabral-Lilly et al. 1997) established the position and orientation of TnT1 fragment binding in parallel with tropomyosin. It is likely that TnT1 plays a part in stabilising the overlap zone between two tropomyosin 
Fig. 8 The binding of the skeletal muscle TnI switch peptide, helix3 (green), to the hydrophobic patch of skTnC- $\mathrm{Ca}^{2+}$ (red). The switch peptide is an amphipathic helix with hydrophobic residue on the face interacting with TnC (Ala118, Met121, Leu122, Leu125, Leu126). Rendered from Vinogradova's X-ray structure 1ytz with segments of TnI C-terminal to Helix 3 and $\mathrm{TnT}$ are removed for clarity. (Color figure online)

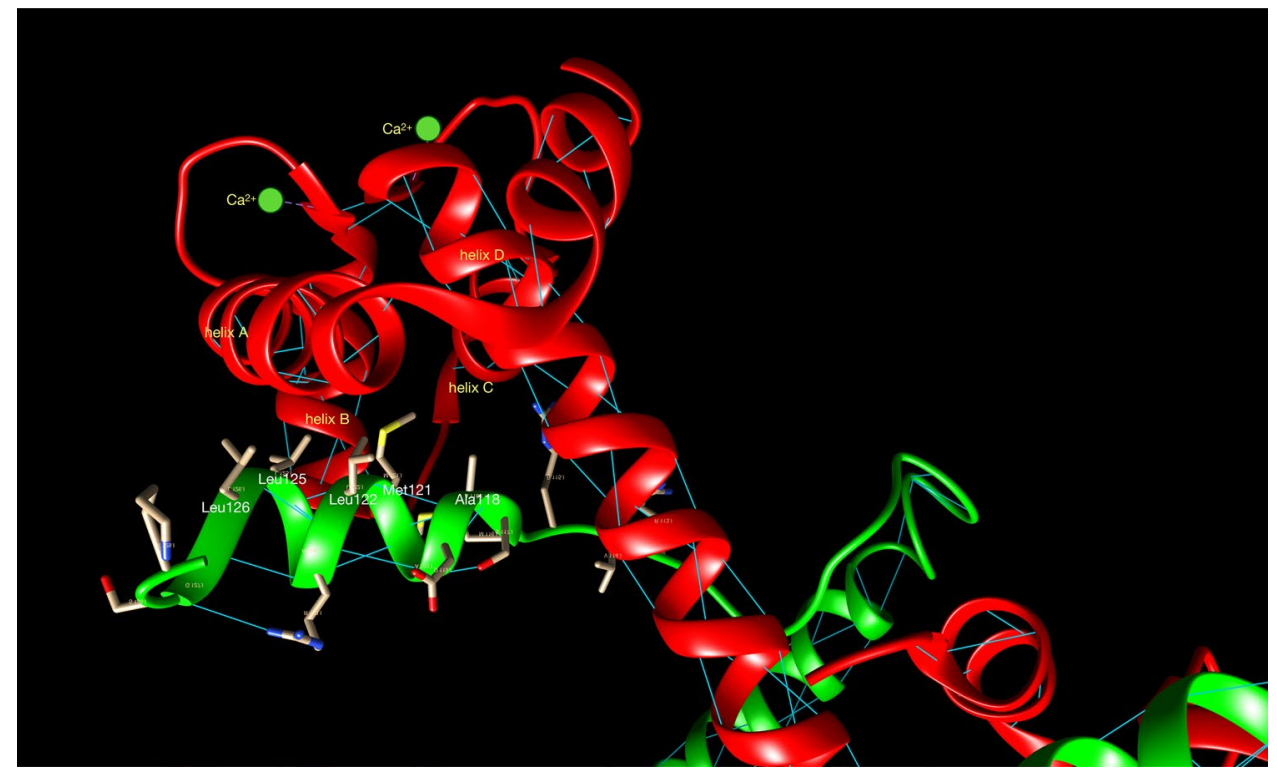

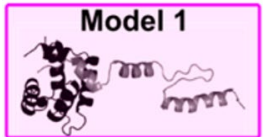
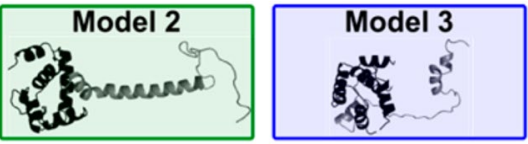

Fig. 9 Three models of the structure of the TnI C-terminal domain with the preceding switch peptide docked on $\mathrm{TnC} \mathrm{Ca}^{2+}$ (left). Model 1 is (Murakami et al. 2005) (NMR, NOE), model 2 is (Blumenschein et al. 2006) (NMR, CSI) and model 3 is (Takeda et al. 2003). From (Metskas and Rhoades 2015), with permission

Table 3 The domains of troponin T, standard numbering for cTnT isoform 6 (P45379-6)

\begin{tabular}{cc}
\hline Residues & Name \\
\hline $1-168$ & T1 domain \\
$169-200$ & Linker peptide \\
$201-288$ & T2 domain \\
\hline $201-225$ & Helix H1 \\
$226-277$ & Helix H2 \\
$278-288$ & C terminal peptide \\
\hline
\end{tabular}

molecules (Murakami et al. 2008; Palm et al. 2001). Functionally, the presence of TnT1 in the thin filament independently enhances the cooperativity of the $\mathrm{Ca}^{2+}$ switch, presumably via its interactions with tropomyosin (Schaertl et al. 1995) (Fig. 10).

There is a flexible linker between TnT1 and TnT2. This is essential for locating and anchoring the troponin complex onto the actin -tropomyosin filament independent of $\left[\mathrm{Ca}^{2+}\right]$. It appears to be largely disordered, however, Helix
$\mathrm{H} 1$ of TnT in the crystal structure may be considered as the C-terminal end of this domain. Both the tropomyosin overlap zone and the linker peptide with Helix H1 are hot spots for mutations that cause cardiomyopathies, indicating the role of these regions in modulating the troponin switch mechanism despite being outside the troponin core. The possible structure of TnT1 and the link peptide interacting with tropomyosin has been largely determined by modelling studies, for example, Fig. 11 and, for now, remains hypothetical. (Manning et al. 2012; Orzechowski et al. 2015).

The TnT2 domain is upstream of the linker region and forms an integral part of the troponin core (residues 226 to 271) (Fig. 10). TnT Helix H2 is the central component of the IT arm where it forms an antiparallel coiled-coil with TnI Helix $\mathrm{H} 2$ that spans the length of troponin and also interacts with TnI helix H1. At the end of TnT helix H2, there are interactions with the $\mathrm{C}$ terminal lobe of $\mathrm{cTnC}$. The last sixteen residues of CcTnT were not resolved in the structure of Takeda et al. (2003), indicating their flexibility. They have functional relevance to troponin since an HCM mutation leading to truncation of the last 14 amino acids is a well-known cause of hypertrophic cardiomyopathy (Franklin et al. 2012; Messer et al. 2016; Thierfelder et al. 1994). In their complete model of the human cardiac muscle troponin core, Zamora et al. used molecular dynamics to assess its conformation and found that the $\mathrm{C}$ terminal 16 amino acids are commonly unstructured and can interact with the TnI 'inhibitory' and N-terminal peptides in the hinge region of troponin or, less frequently, with TnT Helix 2 and C-terminal domain of TnC (Zamora et al. 2016) (Fig. 12). 
Fig. 10 The cTnT2 domain with its relevant structural regions highlighted. Subunits cTnC and cTnI are transparent for clarity. Residues 1 to 201 of cTnT are not present in this structure
Fig. 11 Model of troponin $\mathrm{T}$ and its interaction with tropomyosin and troponin I. WT average structure (grey) with regions that are structurally sensitive to TNT1 mutations highlighted. The highlighted regions are colored as a function of their subunit: yellow, cTnT; blue, cTnI; and red, cTnC. From (Manning et al. 2012) with permission. (Color figure online)
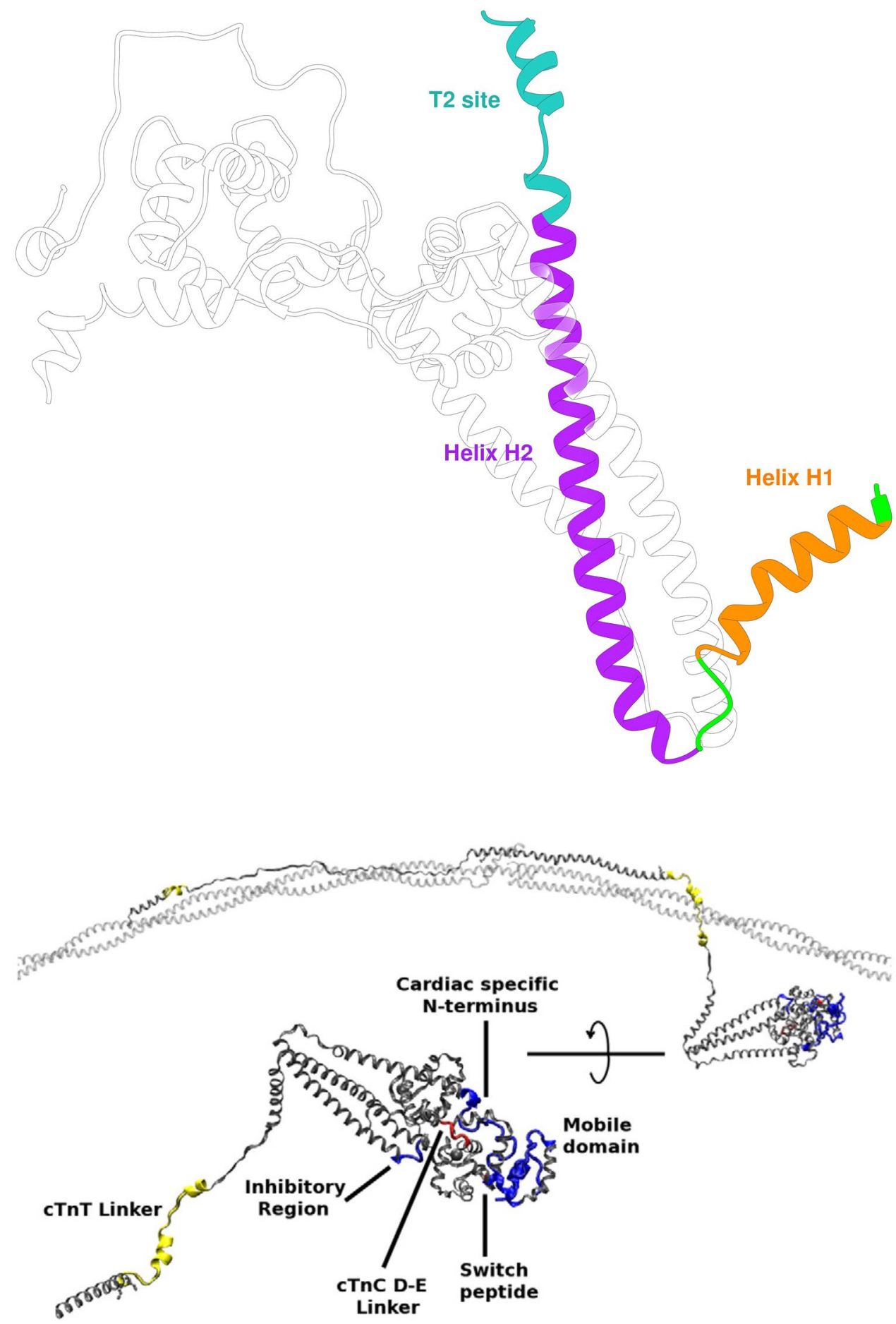

\section{Troponin in the thin filament}

Whilst the isolated troponin molecule is a suitable subject for detailed structural analysis, in vivo troponin is a fully integrated component of the thin filament along with tropomyosin and actin. Troponin's interactions with actin and tropomyosin are the basis of $\mathrm{Ca}^{2+}$-dependent regulation of the thin filament (Gordon et al. 1997) and all the measurements of troponin regulatory function involve the whole thin filament interacting with myosin. The fundamental structure of the thin filament is the double helix of actin monomers (13 per full turn). The elongated tropomyosin molecule follows the actin helix at a ratio of $1 \mathrm{Tm} / 7$ actin and the tropomyosin molecules are joined end-to-end producing a continuous strand. One troponin 


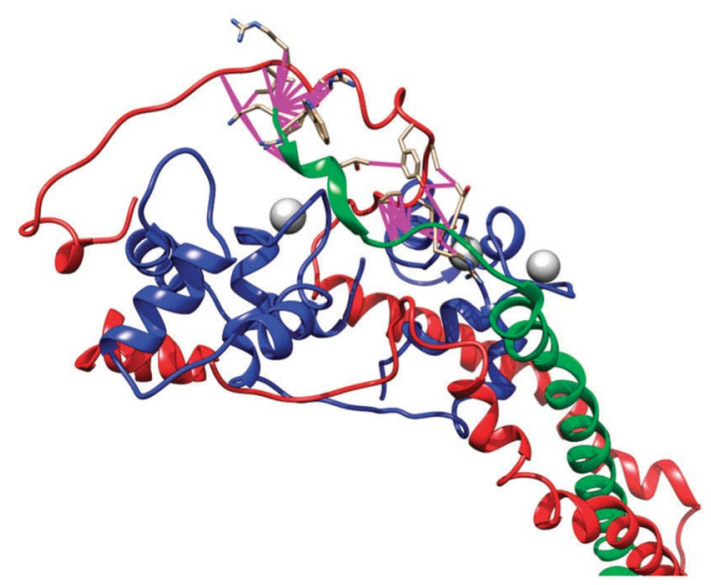

Fig. 12 The two most probable conformations of the C-terminus of troponin $\mathrm{T}$ determined by molecular dynamics simulations (Zamora et al. 2016). Left is the more common configuration. TnT is in green,

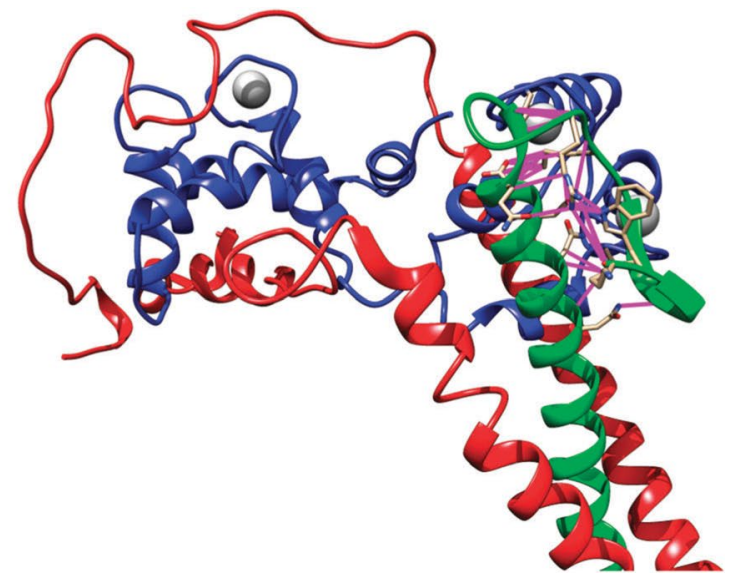

magenta bars represent hydrogen bonding between $\mathrm{TnT}$ and $\mathrm{TnI}$ or TnC. (Color figure online)

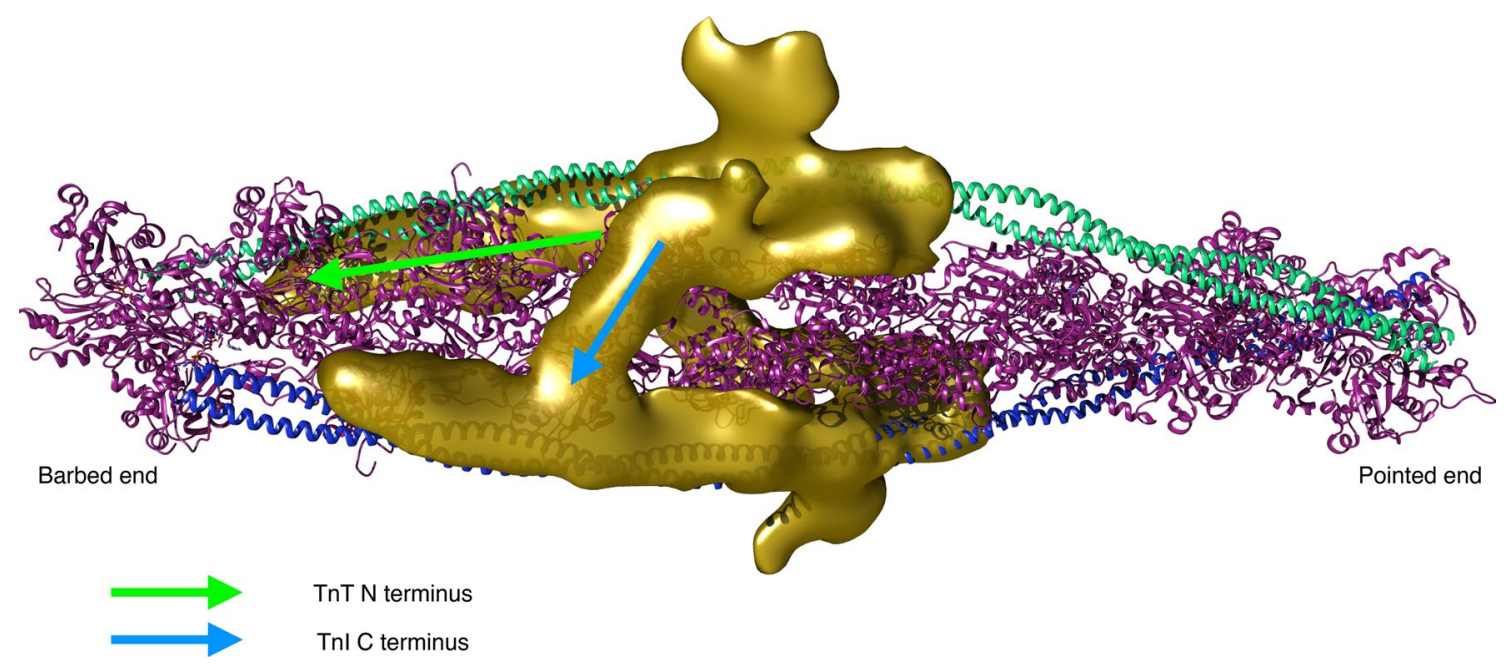

Fig. 13 Troponin rendering onto the thin filament. The thin filament is represented by two coiled-coil tropomyosin monomers shown in cyan and blue over a core of actin monomers shown in ribbon view in magenta. The calculated troponin electron density is represented

is located on the thin filament per tropomyosin (i.e. per 7 actins). Troponin-tropomyosin in the two strands of actin helix are orientated in parallel with their ends in register. The interactions between actin, tropomyosin and troponin in one strand of the helix are fundamental but additional interactions may be possible across the filament, since two troponins are bound at nearly the same level.

Thin filament structure has mainly been studied by electron microscopy and fibre X-ray diffraction (Lehman 2016). A recent model of troponin orientation and position in the thin filament in the absence of $\mathrm{Ca}^{2+}$ has been achieved using electron microscopy by Yang et al. (2014). This combines the electron density envelope from electron microscopy, in gold. The image data was kindly supplied by Dr William Lehman (Boston University, MA, USA) based on Yang et al. (2014). Image modified from Papadaki and Marston (2016) with permission

fibre X-ray diffraction (Poole et al. 2006) and X-ray crystallography structures of the component proteins with routines for fitting the structures into the electron density envelope. For actin and tropomyosin in the active and relaxed states the structures are well established whereas for troponin the fitting is still controversial and the low resolution allows for multiple possible fits. In Yang et al's interpretation, the longitudinal element parallel with tropomyosin would be the $\mathrm{T} 1$ domain of troponin $\mathrm{T}$. One of the more striking elements of these reconstructions is the 'bar' reaching across the filament (Fig. 13). The reconstruction is in the absence of $\mathrm{Ca}^{2+}$ and so likely shows how the $\mathrm{C}$ terminus of $\mathrm{TnI}$ reaches across actin and binds near tropomyosin in the blocked state. 
Fig. 14 The barbed end (Zband end) of the actin filament is on the left of the figure. Difference density maps calculated by subtracting docked F-actin (grey) and tropomyosin (orange) models from the single particle reconstructions leaving density attributable only to troponin (blue). Filament shown in two orientations rotated by $90^{\circ}$. The lower orientation approximates to the orientation in Figs. 1, 13 and 16. From (Paul et al. 2017) with permission. (Color figure online)
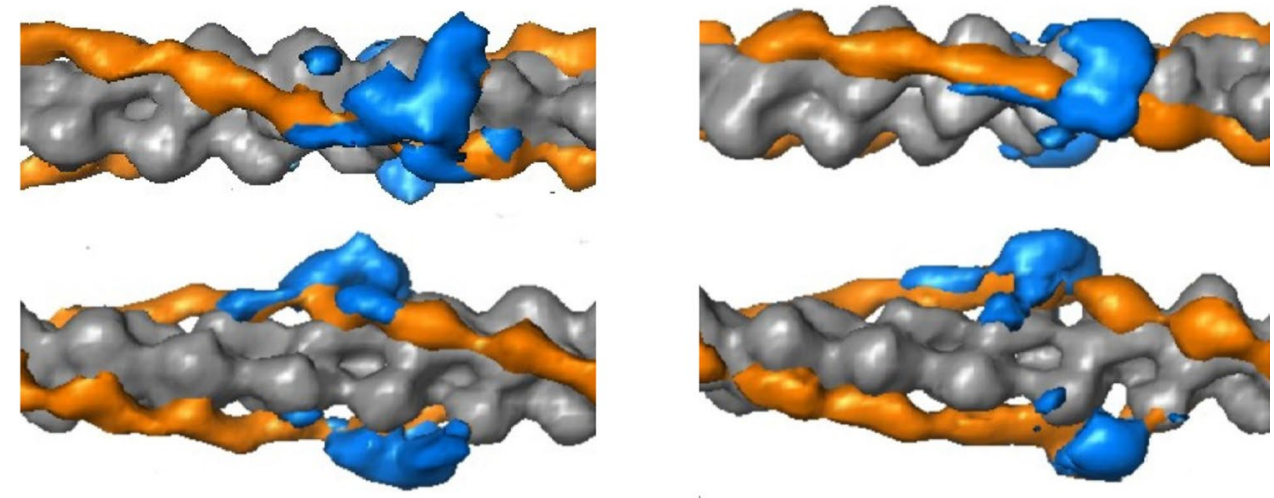

$+\mathrm{Ca}^{2+}$

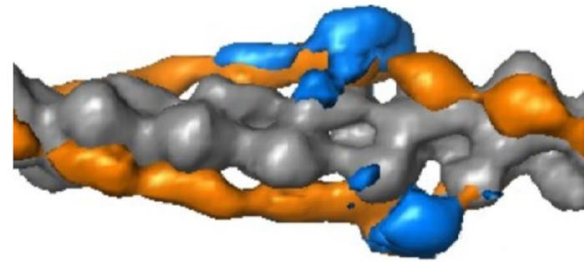

$-\mathrm{Ca}^{2+}$
Another model, produced by Paul et al. is proposed to be more correct since it uses a non model-based single particle analysis of the electron micrographs (see Fig. 1). The main features are the same but, interestingly, this model shows significant differences in the location of the putative Tn mass on the thin filament in the presence and absence of $\mathrm{Ca}^{2+}$ and also fits the troponin core $\mathrm{X}$-ray structure into the electron density envelope differently (Paul et al. 2017) (Fig. 14).

All electron microscopy-based studies suffer from the problem that they only visualize a static state of the thin filament and that the visualization procedures (negative stain or cryopreservation) may change the thin filament structure. Since the thin filament is a dynamic system, methodology that can measure dynamics are needed. Recently, Sun et al. have used fluorescence polarization of probes fixed on troponin to directly determine the orientation of the Tn complex and the effect of $\mathrm{Ca}^{2+}$ within a contracting muscle (Knowles et al. 2012; Sevrieva et al. 2014). The overall orientation of troponin agrees with the Yang model, although the orientation of the IT is more slewed across the filament. Importantly, the IT arm barely changes in the presence of $\mathrm{Ca}^{2+}$, whilst the $\mathrm{TnC} \mathrm{N}$-terminal domain orientation does. NTnC is located close to the actin filament and yet it is capable of substantial hinge movement: three interconverting metastable conformations were calculated in the steady state. Figure 15 shows the three conformations superimposed with $\mathrm{A} 1$ predominating in the absence of $\mathrm{Ca}^{2+}$ and $\mathrm{A} 3$ being favoured when $\mathrm{Ca}^{2+}$ is bound.

An alternative approach is through computational chemistry. Full atomistic simulation of the complete thin filament structure has been achieved by Schwarz (Williams et al. 2016) through the positioning of troponin on the actin filament using docking calculations. The model of the thin filament is based on individual protein structures and known relationships, as described above, and then refined to avoid atom-atom clashes followed by iterative calculations of a best structure by energy minimization. Such a model may then be developed in more detail using molecular

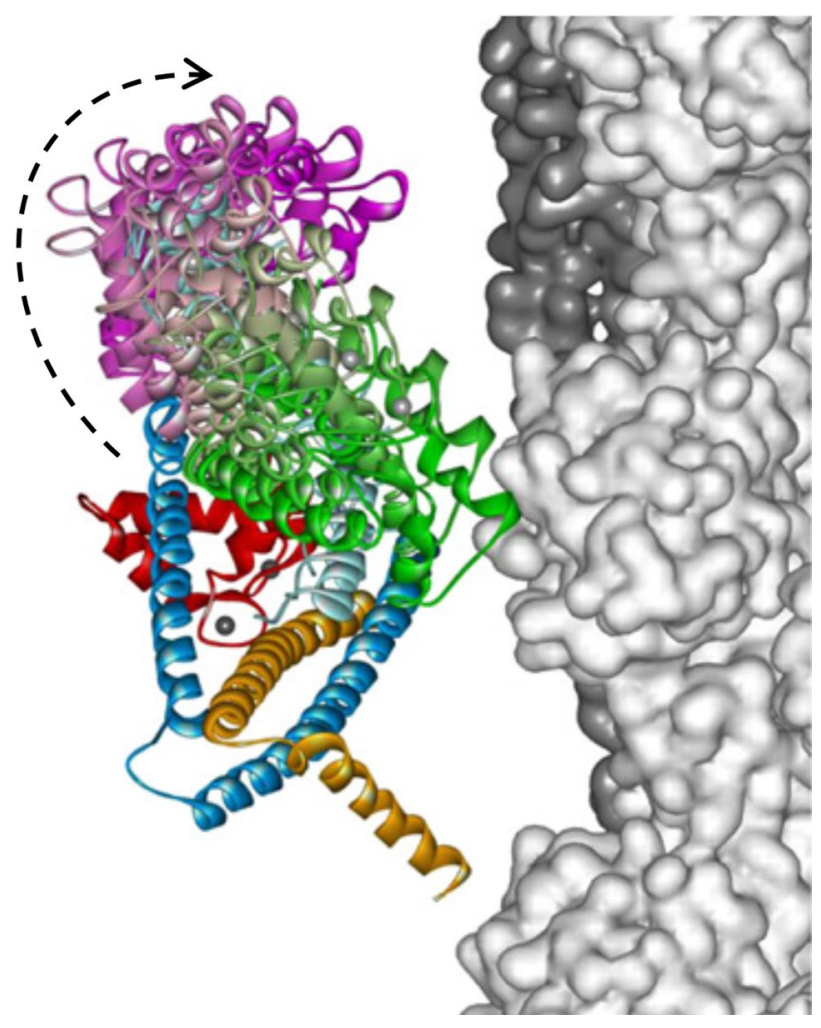

Fig. 15 Troponin orientation on actin determined from fluorescence polarization measurements. The three metastable positions of the $\mathrm{N}$-terminal domain are superimposed: green, A1, light purple, A2 and magenta, A3. The arrow indicates the transition from relaxed to active $\left(+\mathrm{Ca}^{2+}\right)$ contraction (Sevrieva et al. 2014) with permission. (Color figure online)

dynamics simulations although with such a large ensemble (28,847 atoms plus water) only short simulation times (totaling $<10 \mathrm{~ns}$ ) have been so far achieved by this group (Fig. 16). Experience with atomistic simulations of the troponin core indicate that a steady state is reached only after about $100 \mathrm{~ns}$ and that the disordered parts of the structure do not reach convergence on any structure, even after $10 \mathrm{~s}$ 


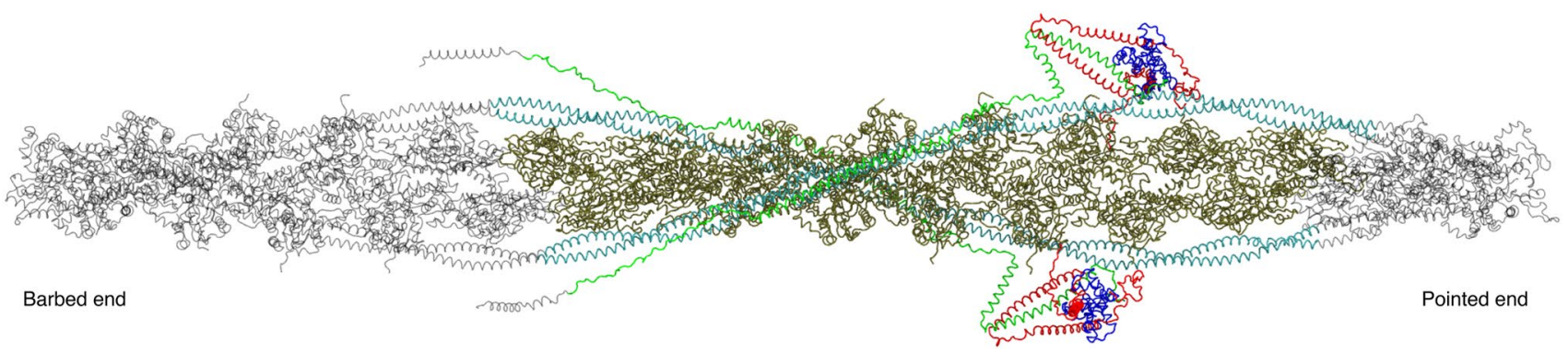

Fig. 16 Model of the thin filament built by Williams et al. based on computational chemistry. (Williams et al. 2016) in grey. The shorter model of Gould and Zamora is coloured. (Color figure online)

of $\mu$ s, indicating this structure is provisional (Zamora et al. 2016; Zamora 2019).

Gould and Zamora have developed a smaller model system ( 15 vs. 25 actins long) with restoration of native sequences, separate + and $-\mathrm{Ca}^{2+}$ simulations and different computational methods to obtain simulations of at least a microsecond which has the potential for providing an analytical solution to troponin regulatory structure and dynamics changes in the thin filament comparable with that already done with troponin (Zamora 2019).

\section{How the $\mathrm{Ca}^{2+}$ switch works at the atomic level}

The mechanism by which troponin switches the activity of the thin filament in response to $\mathrm{Ca}^{2+}$ has been established for some time. At low cytosolic $\mathrm{Ca}^{2+}$ levels, the formation of the actomyosin complex is sterically inhibited by Troponin I (TnI), through its $\mathrm{C}$ terminus binding to actin and locking tropomyosin in a blocking position such that strong binding of actin to myosin is not allowed. When the levels of $\mathrm{Ca}^{2+}$ increase, a single $\mathrm{Ca}^{2+}$ ion binds to the regulatory $\mathrm{N}$-terminal cTnC site II. It provokes an intra-molecular conformational shift, opening up a hydrophobic patch of $\mathrm{NcTnC}$ and exposing it for interaction with the cTnI switch peptide, which is also associated with a hinge motion of the $\mathrm{N}$-terminal $\mathrm{TnC}$ domain relative to the IT domain (Figs. 5, $8,14)$. Binding of the TnI switch peptide to the hydrophobic patch, in turn, pulls the $\mathrm{C}$ terminus of TnI away from actin. This permits a cooperative shift of the tropomyosin molecule across the actin surface, exposing all the myosin binding sites on actin and thus permitting cross-bridge cycling.

This has been incorporated into structural models, three of which are shown in Fig. 17; the fundamental mechanism has not changed, but with each iteration more detail has been added. The model by Malnic et al. (1998) shows the state of the art achieved by site-directed mutagenesis studies on the troponin complex. The fundamental $\mathrm{Ca}^{2+}$ switching is achieved by the switch of $\mathrm{TnI} \mathrm{C}$ terminus binding from actin, where it inhibits, to the troponin $\mathrm{C}$ hydrophobic patch when $\mathrm{Ca}^{2+}$ is bound.

The model of (Takeda et al. 2003) incorporates this into the 3D model of troponin obtained by X-ray diffraction; with the precise interacting sequences identified.

The final model from Solaro and Kobayashi (2011) now incorporates the whole thin filament into the scheme; in this case the known interactions between the regulatory proteins are all accounted for although the structural arrangements remain speculative.

Models like these are based on fixed structures and do not represent the dynamic nature of the thin filament. This is an area that has begun to be tackled. Lehman and Orzechowsky have considered the regulatory conformational changes of the 'steric blocking' mechanism of actin-tropomyosin in terms of an energy landscape in which tropomyosin is located in a set of energy wells on the actin surface whose relative stability and energy barriers between states is dictated by the regulatory state (open, closed and blocked) controlled by troponin, $\mathrm{Ca}^{2+}$ and myosin heads (Kiani et al. 2018; Orzechowski et al. 2014; Lehman 2017). Likewise, the troponin $\mathrm{Ca}^{2+}$-switch may be represented as a reaction coordinate where the probability and rates of progress between multiple states from inactive to active is determined by the $\mathrm{Ca}^{2+}$ binding and the associated energy barriers (Dong et al. 1999; Stevens et al. 2017) (Fig. 18).

It is noteworthy that mutations and post-translational modifications have rather small effects on $\mathrm{Ca}^{2+}$-sensitivity and generally do not change average structure, but rather alter the dynamics of the $\mathrm{Ca}^{2+}$ switch resulting in a change in $\mathrm{Ca}^{2+}$-sensitivity. (Dong et al. 2008; Marston et al. 2013; Stevens et al. 2017).

\section{Modulation of the $\mathrm{Ca}^{2+}$ switch by troponin I phosphorylation}

The heart possesses a unique regulation mechanism that allows it to meet increased oxygen demand from the rest of the body. During periods of high stress or increased 


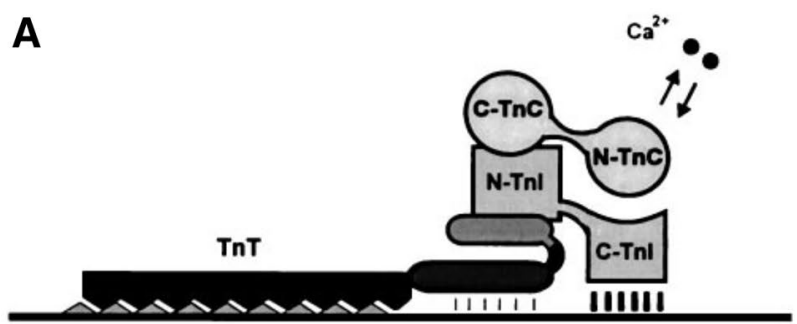

inhibition
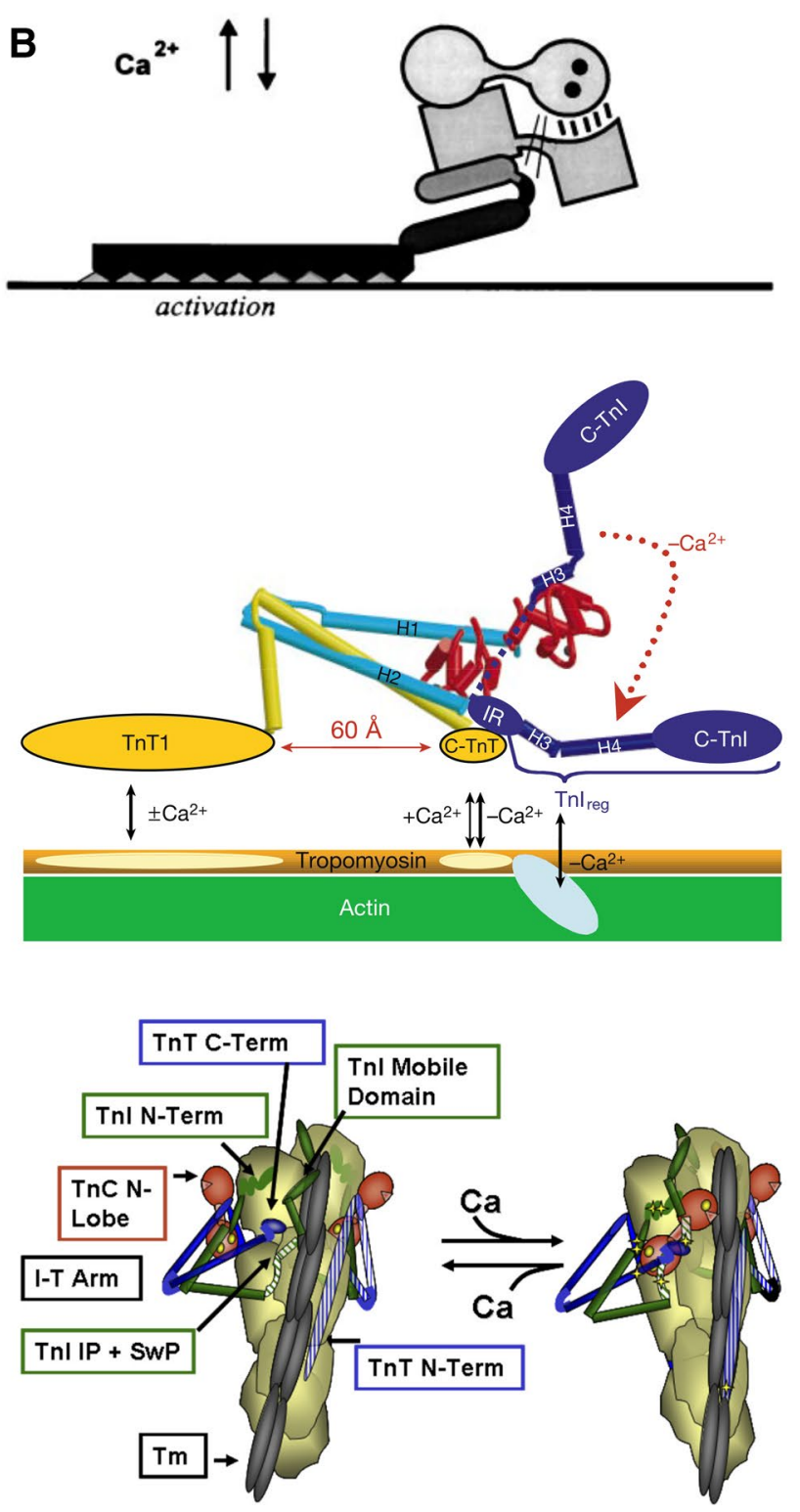

Relaxed

Active

Fig. 17 Structural models describing $\mathrm{Ca}^{2+}$ regulation of thin filaments by troponin

physical activity, catecholamines such as adrenaline and noradrenaline are released into the bloodstream and from sypathetic nerve endings and subsequently bind to $\beta-1$

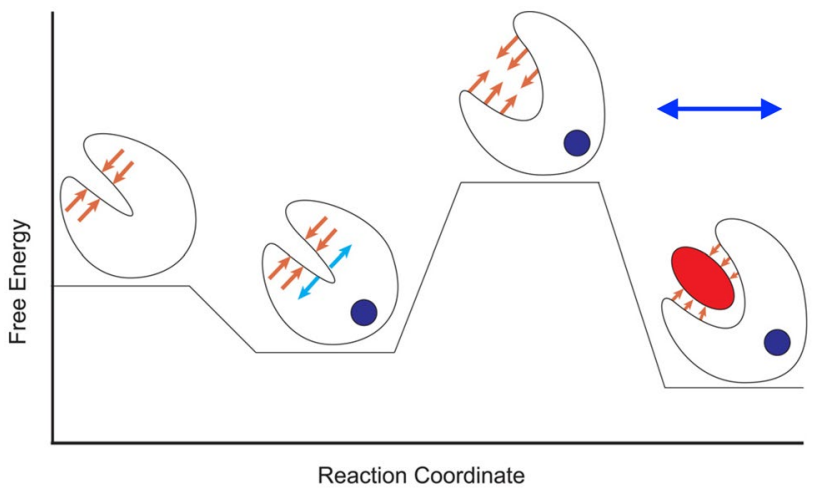

Fig. 18 Schematic of the energetic landscape of N-cTnC activation. $\mathrm{N}$-cTnC is shown as a cartoon. $\mathrm{Ca}^{2+}$ is a blue circle, and the TnI switch peptide is represented as a red ellipse. Lower energy states are more favourable. The orange arrows represent the resistance to the conformational change caused by the hydrophobic cleft. The blue arrows indicate conformational strain introduced by $\mathrm{Ca}^{2+}$ binding. The $\mathrm{Ca}^{2+}$-bound, open conformation relieves the conformational strain while occluding the hydrophobic cleft and is therefore the most favourable conformation. From (Stevens et al. 2017), with permission. The line represents the range of the system accessed by phosphorylation and mutations. (Color figure online)

receptors in cardiac myocytes. This leads to activation of adenyl cyclase via Gs, an increase of the cytosolic cyclic adenosine monophosphate (cAMP) levels and activation of protein kinase A (PKA), which phosphorylates cTnI and other sarcomeric proteins. cTnI is phosphorylated at residues Ser22 and Ser23 in the N-terminal peptide (residues 1 to 36), which is exclusive to the cTn isoform (Al-Hillawi et al. 1995; Ayaz-Guner et al. 2009; Mittmann et al. 1990). The effect of this post-translational modification is a decrease of affinity of cTnC for $\mathrm{Ca}^{2+}$ due to altered cTnC-cTnI interactions linked with a higher rate of $\mathrm{Ca}^{2+}$ dissociation from cTnC (Dong et al. 2007; Robertson et al. 1982; Solaro et al. 1976). See Fig. 19.

The faster relaxation rate (lusitropy) of the cardiac muscle due to TnI phosphorylation is essential for shortening the heart contraction-relaxation cycle allowing for a faster heart rate.

Although the physiological implications of the modulation of the $\mathrm{Ca}^{2+}$ switch by $\mathrm{TnI}$ phosphorylation are well understood, the underlying structural mechanism by which it occurs has remained elusive. The interaction of the N-terminal phosphorylatable peptide of TnI with TnC has been the centre of attention for studies on this process.

Phosphorylation of $\mathrm{cTnI}$ has negligible effects on the $\mathrm{N}$-domain conformation but it affects $\mathrm{Ca}^{2+}$ sensitivity and modifies the kinetics of opening and closing of the $\mathrm{N}$-domain induced by binding of $\mathrm{Ca}^{2+}$ and $\mathrm{S} 1$ (Dong et al. 2007). A seminal NMR study by Baryshnikova, M. X. Li, and Sykes (Baryshnikova et al. 2008a) on partial fragments of the core region of cTn established that both the 
Fig. 19 Effect of dephosphorylation on mouse myofibril contractility. a The $\mathrm{Ca}^{2+}$-sensitivity curve of isometric force for unphosphorylated myofibrils (open circles, dashed line) is shifted to the left of the phosphorylated myofibrils (solid circles, solid line. b Kinetic parameter $\mathrm{k}_{\mathrm{REL}}$ at maximally activating $\mathrm{Ca}^{2+}$ and SL $2.17 \mu \mathrm{m}$. From Vikhorev et al. (2014)
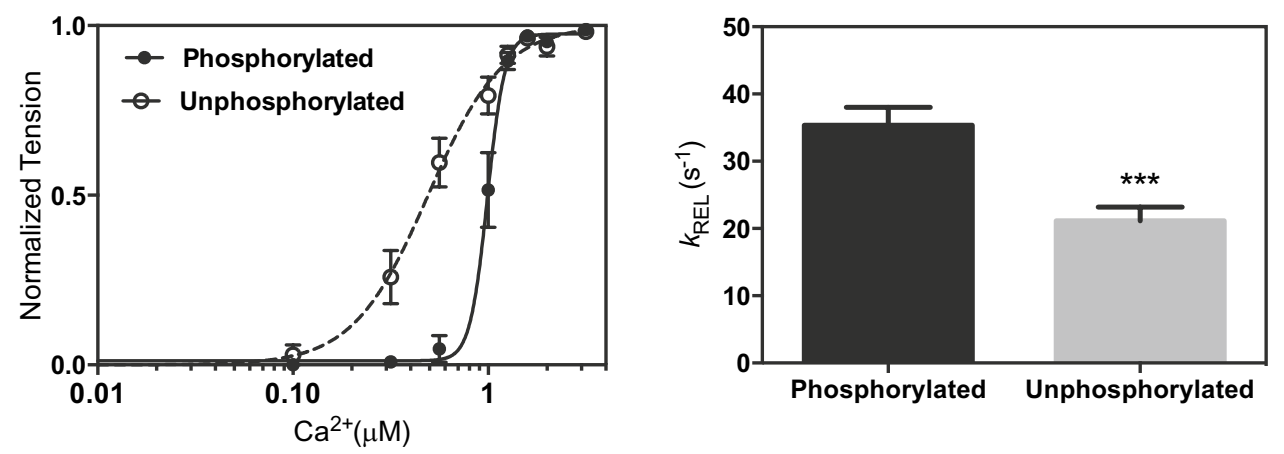

$\mathrm{N}$-terminus of $\mathrm{TnI}(1-33, \mathrm{NcTnI})$ and the switch peptide simultaneously bind to the $\mathrm{N}$-terminal domain of $\mathrm{TnC}$ (NcTnC) (see Table 2). The switch peptide was found to bind to NcTnC in the same region regardless of the presence of NcTnI in both unphosphorylated (uP) and phosphorylated (P) states, but in the absence of NcTnI this interaction is tighter $\left(K_{d}=154 \mu \mathrm{M}\right)$. Additionally, the binding of NcTnI to NcTnC was found to be more efficient in the uP state, and that phosphorylation created no new conformational changes to NcTnC. Interestingly, this study reported that the phosphorylation signal provokes an increased strength of interaction between the cTnI switch peptide and $\mathrm{NcTnC}$ (in the P state, $K_{d}=370 \pm 30 \mu \mathrm{M}$ compared to $K_{d}=600 \pm 100 \mu \mathrm{M}$ in the uP state) rather than by a direct alteration of the $\mathrm{Ca}^{2+}$ interaction with $\mathrm{cTnC}$ in its binding pocket, EF-hand II $\left(K_{d C a}=5 \pm 3 \mu \mathrm{M}\right.$ in both the $\mathrm{P}$ and $\mathrm{uP}$ states). This two-fold difference between $\mathrm{uP}$ and $\mathrm{P}$ cTn in the switch peptide-NcTnC affinity of interaction is comparable with experiments in whole thin filaments and can account for the differences in relaxation rates of the thin filament. In fact, Baryshnikova et al. point out that the ordering of cTnI's switch peptide- NcTnC strength of interactions match with the inverse order of the relaxation speed of myofibrils under different physiological conditions: no NcTnI $>$ NcTnI P $>$ NcTnI uP.

This behaviour is comparable with other studies on enzyme regulation by phosphorylation where several unifying themes have been proposed (Colson et al. 2012). Firstly, the effect of phosphorylation changes the structure of the bound complex without dissociation, secondly that phosphorylation induces transitions between order and dynamic disorder (or changes in the magnitude of disorder), as demonstrated by Hwang for TnI-TnC (Hwang et al. 2014) and, finally, structural states are only loosely coupled to phosphorylation; i.e., complete phosphorylation induces dramatic functional effects with only a limited shift in the equilibrium between ordered and disordered. In terms of the troponin $\mathrm{Ca}^{2+}$ switch, the phosphorylation effects are limited to affecting the kinetics and equilibrium of the $\mathrm{TnI} / \mathrm{Ca}^{2+}$ / open (active) configuration of troponin $\mathrm{C}$ (right hand end of Fig. 18) that is separated from the rest of the activation pathway by a large energy barrier.

Recently computational molecular modelling and molecular dynamics simulations have become the method of choice for understanding this system. The first MD study of the structural effects of cTnI phosphorylation was performed by (Cheng et al. 2014, 2015). This study used a reasonably complete structure of the core domain of $\mathrm{cTn}$ : the complete sequence of cTnC (residues 1 to 161), cTnI (residues 1 to 172) and cTnT (residues 236 to 285). The authors compared unphosphorylated cTn to pseudophosphorylated cTn using a double phosphomimic substitution: cTnI $\mathrm{S} 23 \mathrm{D} / \mathrm{S} 24 \mathrm{D}$. The main observations were the formation of a new interaction between NcTnI and the 'inhibitory' peptide after pseudo- phosphorylation and an overall increase in the system's fluctuation profiles. This observation was similar to an NMR study on the conformational effects of cTnI phosphorylation by Howarth et al. (2007). It was proposed that phosphorylation caused an extension of a helical motif at the end of the NcTnI region which reduced the interactions between NcTnC and NcTnI. Thus, the first few acidic residues of $\mathrm{NcTnI}$ were positioned to interact with the positively-charged 'inhibitory' peptide of cTnI, defining a new and potentially significant interaction in troponin.

A more extensive study by Zamora et al. (2016) replicated the simulations of Cheng et al., using a complete model of the core domain of cTn, an updated force-field and substantially more sampling (both a larger volume simulated and $10 \times$ longer times). This study failed to reproduce the reported effects of phosphorylation on the structure and dynamics of cTn. It was found that the interactions between the NcTnI region and the inhibitory peptide were only occasionally sampled in any phosphorylation state and that there was a lack of convergence between independent simulations despite the longer simulation times. This suggests the results in the original publication could have been random events. Although some apparent phosphorylation-related changes were observed, Zamora et al's main conclusions were that the structural and dynamic changes induced by phosphorylation could not be defined with any statistical significance 
Fig. 20 The three macrostates of troponin located in their average positions on the landscape formed by the first two timestructure Independent Components Analysis. a WT unphosphorylated macrostates and the MFPTs of transition between them. b WT Phosphorylated macrostates and the MFPTs of transition between them. The node sizes reflect the relative stability of the macrostates, the arrow labels indicate the Mean First Passage Times of transition in $\mu$ s (Zamora 2019)
A

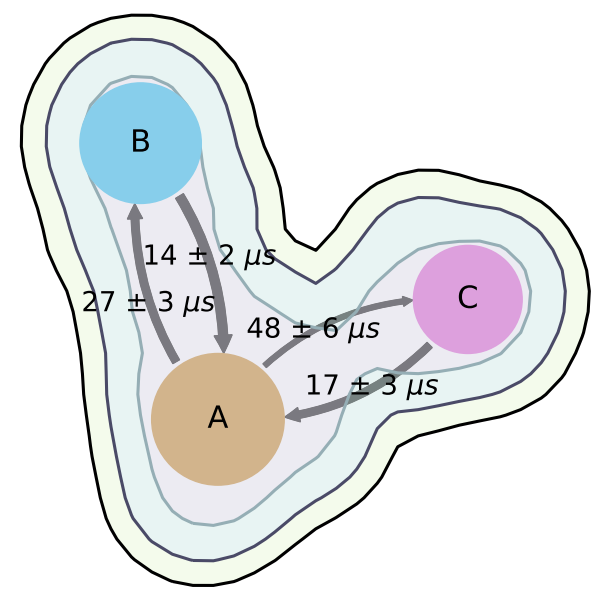

B

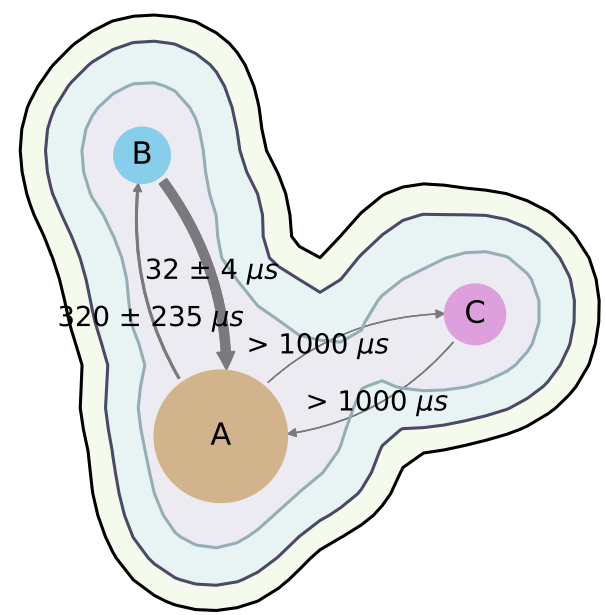

using only a small number of independent MD trajectories a few hundred ns of length.

Recently Zamora et al. have produced and analysed a substantially larger dataset (over $30 \mu$ s simulation of both $\mathrm{P}$ and unP troponin) produced with an updated force field (Zamora 2019). This new force field, termed ff $14 \mathrm{SB}$, belongs to the AMBER protein force fields and represents a revised and updated version of ff99SB (Maier et al. 2015). A complete refit of the side chain dihedral parameters was performed. Overall, this improved secondary structure predictions and NMR scalar couplings for proteins in solution. Additionally, they used Markov State Models (MSMs) to understand the dynamic transitions between the structural ensembles of cTn. This revealed a significant difference between phosphorylated and unphosphorylated troponin (Fig. 20).

MSMs are a suitable technique to analyse MD simulations since they model the transitions between a set of disjoint states as a memoryless network. The mathematical underpinnings of this analysis technique have been known for a long time (Husic and Pande 2018). However, their applicability to the study of complex biomolecular processes has experienced a surge of popularity thanks to improvements in the estimation of these models and in the increased capabilities of generation of simulated dynamical data into timescales of biological relevance (Prinz et al. 2011). MSMs require the partition of the structural phase space into hundreds, or even thousands, of microstates to provide a highly detailed description of the conformational dynamics in multidimensional space. The resulting models are thus very complex: to aid in their interpretability, it is often desirable to derive from them simple kinetic models with la ow number of states. The objective is to obtain a handful of metastable macrostates formed by MSM microstates which interconvert quickly between them. Based on the spectral gap that is observed in the timescales, Zamora et al. decided to partition of the space into three macrostates.
The relative population of the macrostates at equilibrium and their interconversion rates are altered upon phosphorylation.

The simulation data may also be interpreted in terms of the current models for the $\mathrm{Ca}^{2+}$-switch. Zamora et al. followed the model of Kekenes-Huskey et al. (2012) (Fig. 18). Because the simulations are in the $\mathrm{Ca}^{2+}$-activated state, the cTnI switch peptide is always bound to the hydrophobic patch and was never observed to unbind in any of the simulations.

The cTnC helix A-B interhelical angle was calculated as a function of time. Both unphosphorylated and the phosphorylated states remained largely in the open conformation, but, transitions to the closed conformation were observed to be more frequent in the WT apo unphosphorylated state. From time-series data, it was possible to estimate the freeenergy barrier associated to this conformational rearrangement, as well as the mean first passage time (MFPT) to go from an open to closed conformation. It was observed that the energy barrier associated for the open-to-close conformation of the hydrophobic patch of cTnC is lower for the unphosphorylated state than for the phosphorylated state, with a $\Delta \Delta G_{u P \rightarrow P} \approx 27 \mathrm{kcal} \mathrm{mol}^{-1}$. Furthermore, the maximal angle that is observed for the unphosphorylated state is $141^{\circ}$, whilst the maximal angles that are achieved in the phosphorylated simulations is $119^{\circ}$. Finally, the kinetics of closing are also altered, since the MFPT between the closed and open states is on the low microsecond time regime in the unphosphorylated state, whilst it is increased by an estimated order of magnitude in the phosphorylated state, suggesting phosphorylation enhances the probability of the open state.

In addition helicity was investigated throughout the troponin sequence. The average change in the propensity to form a randomly coiled structure was mapped onto each residue of the cTn core domain. As can be appreciated, a large portion of cTn is unaltered upon phosphorylation. Of the residues that are changed, the I36-V72 pair were 


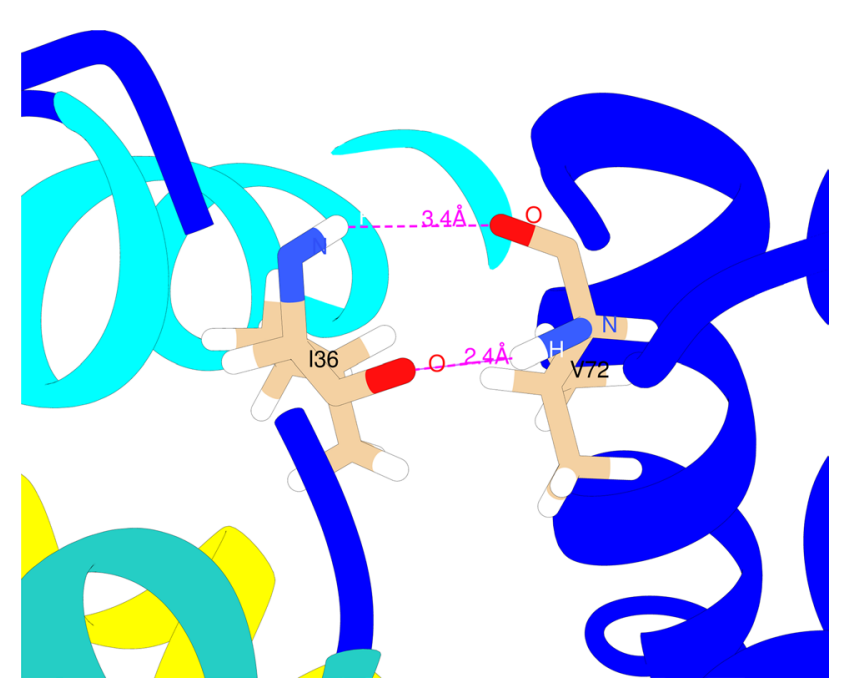

Fig. 21 The two backbone hydrogen bonds that can be formed between cTnC I36 and V72 in the open state and that are modulated by phosphorylation

of particular interest since they are not sequential but they are spatially close. I36 and V72 are located in the loops connecting helices $\mathrm{A}-\mathrm{B}$ and $\mathrm{C}-\mathrm{D}$, respectively, and can interact through backbone hydrogen bonds in the open state (see Figs. 5, 6 and 21). Thus, it was hypothesised that the increased frequency of isolated $\beta$ bridge formation between I 36 and V72 could be the reason behind the lower frequency of conformational change that is observed in the NcTnC hydrophobic path in the phosphorylated states. Additionally, V28, L29 and G40 are located at the end of helix A: their propensity towards disorder in the phosphorylated state could also contribute to this mechanism-a less rigid helix could have a harder time pivoting towards the closed conformation of the hydrophobic patch. It was calculated that phosphorylation triggers an increase of about $10 \%$ in the cluster population corresponding to having both hydrogen bonds present. Interestingly, not only are the relative populations of the hydrogen bonds altered by phosphorylation, but also the timescales associated with the creation and dissociation of the bonds. Overall these simulations indicate that phosphorylation stabilises the open state, in a similar way to that proposed by Barashnikov et al. (2008a).

The model derived from molecular dynamics offers an explanation as to how mutations could uncouple TnI phosphorylation from the change in $\mathrm{Ca}^{2+}$-sensitivity, a phenomenon seen with many HCM and DCM-related mutations (Memo et al. 2013; Messer et al. 2016; 2017). Early studies indicate that mutations destabilise the open state (Zamora 2019).
Modulation of the $\mathrm{Ca}^{2+}$ switch by mutations

A large number of mutations in troponin subunits have been found to be associated with cardiomyopathies and have been extensively studied. Mutations in TNNNT2 and TNNI3 genes are significant causes of hypertrophic cardiomyopathy and dilated cardiomyopathy, whilst mutations in in TNNC1 are rare causes of these cardiomyopathies, Functionally the HCM mutations all seem to increase myofilament $\mathrm{Ca}^{2+-}$ sensitivity by about twofold (Marston 2011, 2016) and this is proposed to be disease-causing (Spudich 2014). In contrast, the effect of DCM mutations on $\mathrm{Ca}^{2+}$ sensitivity is variable (Marston 2011; Memo et al. 2013). In addition, in many HCM mutations and all DCM mutations in troponin subunits so far tested the relationship between $\mathrm{TnI}$ phosphorylation by PKA and decreased $\mathrm{Ca}^{2+-}$ sensitivity is abolished (Dvornikov et al. 2016; Dyer et al. 2009; Messer et al. 2016; Messer and Marston 2014) and this appears to be sufficient to cause DCM in the long term (Fig. 22) (Wilkinson et al. 2015).

Only a few mutations have been studied at the structural level; as would be expected from their small functional effects, the structural effects of mutations are subtle.

The TnI mutation $R 145 \mathrm{~W}$ has been extensively studied since it was reported to be associated with particularly severe HCM or restrictive cardiomyopathy and because it is located in the 'inhibitory' peptide of TnI (Deng et al. 2001; Elliott et al. 2000; Kruger et al. 2005; Lang et al. 2002). It has been established that this mutation increases $\mathrm{Ca}^{2+}$-sensitivity and uncouples TnI phosphorylation from a $\mathrm{Ca}^{2+}$ sensitivity change (Dvornikov et al. 2016). Two molecular dynamics studies of the troponin core have addressed this mutation. The study of Lindert et al. (2015) may have been compromised by the size of the box used for simulation (Papadaki and Marston 2016; Zamora et al. 2016). However, a study by Dvornikov et al. (2016) used a larger box, although simulation times were still short (100 ns). The mutation did not have any global effects but it was found to cause a significant local reduction in the interaction of amino acid 145 with acidic residues in the cTnC C helix (E63, E59, E56) which was not phosphorylation-dependent. How the mutation affects dynamics and the mechanism for increased $\mathrm{Ca}^{2+}$-sensitivity was not apparent from this study.

The $L 29 Q$ mutation in $c T n C$ has also been investigated. This mutation is regarded as an HCM-causing mutation although the clinical and genetic data is not very extensive (Hoffmann et al. 2001). It has been studied by X-ray diffraction, NMR and fluorescence polarisation (Li et al. 2013; Robertson et al. 2015; Zhang et al. 2013). All these methodologies indicate that the mutation did not perturb the overall structure although some local changes were observed. 
A

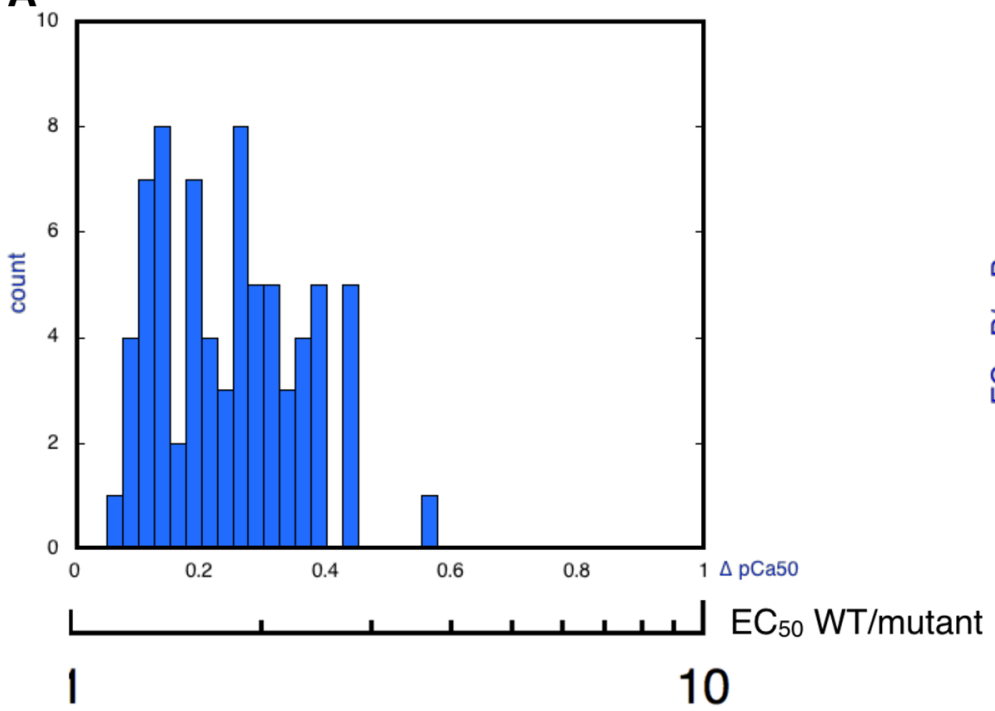

B

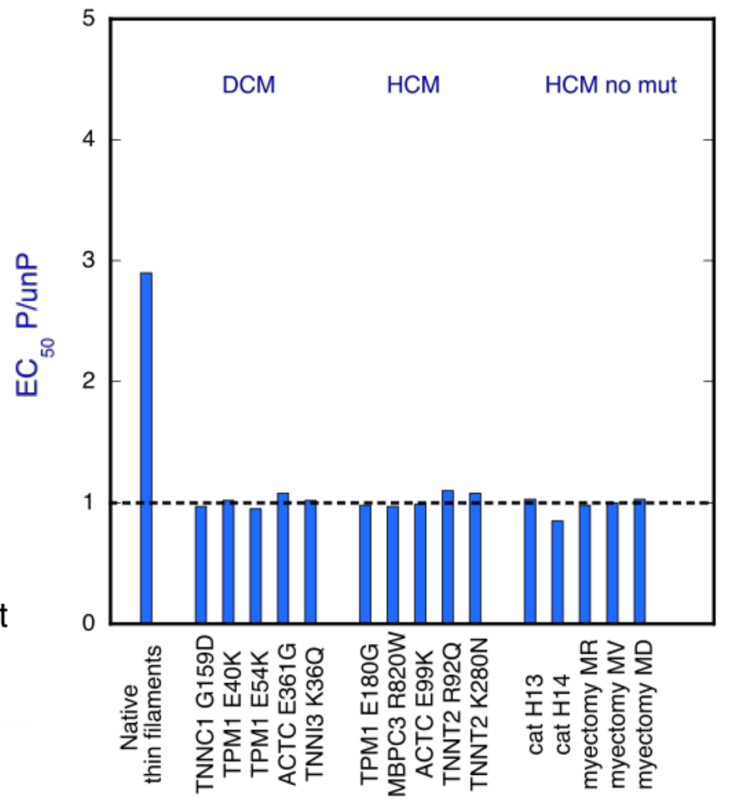

Fig. 22 a Frequency histogram of increase in $\mathrm{Ca}^{2+}$ sensitivity for 71 measurements of HCM mutations compared with wild-type. The plot includes results obtained with 4 measurement methods with 44 mutations in 6 genes. The mean increase in $\mathrm{Ca}^{2+}$-sensitivity is $1.87 \pm 0.07$ fold (sem). From (Marston 2016). b Uncoupling of the relationship

The troponin C G159D mutation is associated with a severe dilated cardiomyopathy and is one of the most thoroughly investigated DCM mutations (Biesiadecki et al. 2007; Dyer et al. 2009; Kaski et al. 2007).

An NMR spectroscopy study by Baryshnikova et al. (2008b) found little difference in structure or dynamics of Mutant TnC, C terminal domain interacting with TnI 34-71 although the affinity of this interaction was reduced from $<1 \mu \mathrm{M}$ in wild-type to $3.0 \mu \mathrm{M}$ in G159D. The significance of this is hard to assess since the structures studied were only fragments of troponin and did not include the regulatory head of troponin or TnT.

Zamora and Gould have analysed the G159D mutation in their model of the complete troponin core in both phosphorylated and unphosphorylated states. Remarkably, simple RMSF plots indicate that phosphorylated G159D is significantly less mobile (a generalised reduction in all three subunits) compared with unphosphorylated G159D and wild-type, phosphorylated or unphosphorylated, which were all similar. This indicates that Phosphorylated G159D is uniquely more rigid. This is reflected in calculations of the helix $\mathrm{A} / \mathrm{B}$ angle where a significant population of more closed angles was observed in phosphorylated G159D, opposite to the effect of phosphorylation on wild-type. This suggests a decreased probability of the open state and may account for the lack of a decrease in $\mathrm{Ca}^{2+}$-sensitivity coupled to phosphorylation in G159D (Zamora 2019). between cTnI phosphorylation and myofilament $\mathrm{Ca}^{2+}$-sensitivity. The ratio of $\mathrm{EC}_{50}$ phosphorylated: Unphosphorylated is 2.4. In wildtype, but 1.0 for the HCM and DCM mutations shown on the x-axis, indicating uncoupling. From (Sheehan et al. 2018)

Kekenes-Huskey et al. examined a number of mutations that affect $\mathrm{Ca}^{2+}$ affinity in a model of $\mathrm{NcTnC}$ and noted that helicity and/or the A/B helix angle dynamics could be altered (Kekenes-Huskey et al. 2012). Recently Stevens et al. conducted an extensive molecular dynamics study of $6 \mathrm{HCM}$ -linked mutations in the $\mathrm{N}$ terminal domain of TnC including L29Q (Stevens et al. 2017). The mutations modified the structural dynamics of $\mathrm{TnC}$ rather than the regulatory $\mathrm{Ca}^{2+}$-binding site. The changes are observed in the relative favourability of the protein conformations that transduce the contraction signal by destabilizing the closed conformation of N-cTnC, stabilizing the open conformation, or stabilizing the interaction of $\mathrm{NcTnC}$ with the $\mathrm{TnI}$ switch peptide.

In summary, the effects of mutations on troponin seem to mimic the effect of phosphorylation/dephosphorylation in that they modulate the dynamics of the $\mathrm{Ca}^{2+}$ switch elements (Fig. 18). This rather general mechanism may explain how mutations in many different proteins of the sarcomere can produce the same phenotype at the molecular level.

\section{Conclusions}

Recent investigations of troponin have confirmed the basic mechanism of the $\mathrm{Ca}^{2+}$-switch, established over the last 20 years and have added structural detail. Present studies are mainly directed towards the subtle modulation of the 
switch by phosphorylation and mutations. These do not produce detectable changes in the steady state but in both cases significant changes in troponin dynamics, exemplified by the A/B helix angle, can be demonstrated by NMR and molecular dynamics simulations. Future studies on troponin structure and function will need to address in detail the intrinsically disordered parts of troponin that play an essential role in both the $\mathrm{Ca}^{2+}$ switch and its physiological and pathological modulation.

Acknowledgements JEZ acknowledges financial support from "La Caixa" Foundation, the Institute of Chemical Biology, Imperial College London and the Engineering and Physical Sciences Research Council (EPSRC) UK.

Open Access This article is distributed under the terms of the Creative Commons Attribution 4.0 International License (http://creativeco mmons.org/licenses/by/4.0/), which permits unrestricted use, distribution, and reproduction in any medium, provided you give appropriate credit to the original author(s) and the source, provide a link to the Creative Commons license, and indicate if changes were made.

\section{References}

Al-Hillawi E, Bhandari DG, Trayer HR, Trayer IP (1995) The effects of phosphorylation of cardiac troponin-I on its interactions with actin and troponin-C. Eur J Biochem 228:962-970

Anderson PAW, Malouf NN, Oakeley AE, Pagani ED, Allen PD (1991) Troponin $\mathrm{T}$ isoform expression in humans. A comparison among normal and failing heart, fetal heart and adult and fetal skeletal muscle. Circ Res 69:1226-1233

Ayaz-Guner S, Zhang J, Li L, Walker JW, Ge Y (2009) In vivo phosphorylation site mapping in mouse cardiac troponin i by high resolution top-down electron capture dissociation mass spectrometry: Ser22/23 are the only sites basally phosphorylated. Biochemistry 48:8161-8170

Baryshnikova OK, Li MX, Sykes BD (2008a) Modulation of cardiac troponin $\mathrm{C}$ function by the cardiac-specific $\mathrm{N}$-terminus of troponin I: influence of PKA phosphorylation and involvement in cardiomyopathies. J Mol Biol 375:735-751. https://doi.org/10.1016/j. jmb.2007.10.062

Baryshnikova OK, Robertson IM, Mercier P, Sykes BD (2008b) The dilated cardiomyopathy G159D mutation in cardiac troponin C weakens the anchoring interaction with troponin I. Biochemistry 47:10950-10960. https://doi.org/10.1021/bi801165c

Bayliss CR et al (2012) Myofibrillar $\mathrm{Ca}^{2+}$-sensitivity is uncoupled from troponin i phosphorylation in hypertrophic obstructive cardiomyopathy due to abnormal troponin T. Cardiovasc Res 97:500-508. https://doi.org/10.1093/cvr/cvs322

Biesiadecki BJ, Kobayashi T, Walker JS, John Solaro R, de Tombe PP (2007) The troponin C G159D mutation blunts myofilament desensitization induced by troponin I Ser23/24 phosphorylation. Circ Res 100:1486-1493

Blumenschein TMA, Stone DB, Fletterick RJ, Mendelson RA, Sykes $\mathrm{BD}$ (2006) Dynamics of the C-terminal region of TnI in the troponin complex in solution. Biophys J 90:2436-2444. https://doi. org/10.1529/biophysj.105.076216

Cabral-Lilly D, Tobacman LS, Mehegan JP, Cohen C (1997) Molecular polarity in tropomyosin-troponin T co-crystals. Biophys $\mathrm{J}$ 73:1763-1770
Cheng Y et al (2014) Computational studies of the effect of the S23D/S24D troponin I mutation on cardiac troponin structural dynamics. Biophys J 107:1675-1685. https://doi.org/10.1016/j. bpj.2014.08.008

Cheng Y et al (2015) Troponin I mutations R146G and R21C alter cardiac troponin function, contractile properties and modulation by PKA-mediated phosphorylation. J Biol Chem 290:27749-27766. https://doi.org/10.1074/jbc.M115.683045

Colson BA, Gruber SJ, Thomas DD (2012) Structural dynamics of muscle protein phosphorylation. J Muscle Res Cell Motil 33:419429. https://doi.org/10.1007/s10974-012-9317-6

Deng Y, Schmidtmann A, Redlich A, Westerdorf B, Jaquet K, Thieleczek R (2001) Effects of phosphorylation and mutation R145G on human cardiac troponin I function. Biochemistry 40:14593-14602

Dong WJ et al (1999) Conformation of the regulatory domain of cardiac muscle troponin $\mathrm{C}$ in its complex with cardiac troponin I. J Biol Chem 274:31382-31390

Dong WJ, Jayasundar JJ, An J, Xing J, Cheung HC (2007) Effects of PKA phosphorylation of cardiac troponin I and strong crossbridge on conformational transitions of the $\mathrm{N}$-domain of cardiac troponin $\mathrm{C}$ in regulated thin filaments. Biochemistry 46:9752-9761

Dong WJ et al (2008) Structural kinetics of cardiac troponin C mutants linked to familial hypertrophic and dilated cardiomyopathy in troponin complexes. J Biol Chem 283:3424-3432. https://doi. org/10.1074/jbc.M703822200

Dvornikov AV, Smolin N, Zhang M, Martin JL, Robia SL, de Tombe PP (2016) Restrictive cardiomyopathy Troponin-I R145 W mutation does not perturb myofilament length dependent activation in human cardiac sarcomeres. J Biol Chem 291:21817-21828. https ://doi.org/10.1074/jbc.M116.746172

Dyer E et al (2009) Functional analysis of a unique troponin cC mutation, Gly159Asp that causes familial dilated cardiomyopathy, studied in explanted heart muscle. Circ Heart Fail 2:456-464. https://doi.org/10.1161/CIRCHEARTFAILURE.108.818237

Elliott K, Watkins H, Redwood CS (2000) Altered regulatory properties of human cardiac troponin I mutants that cause hypertrophic cardiomyopathy. J Biol Chem 275:22069-22074

Franklin AJ, Baxley T, Kobayashi T, Chalovich JM (2012) The C-terminus of troponin $\mathrm{T}$ is essential for maintaining the inactive state of regulated actin. Biophys J 102:2536-2544. https://doi. org/10.1016/j.bpj.2012.04.037

Gordon AM, LaMadrid MA, Chen Y, Luo Z, Chase PB (1997) Calcium regulation of skeletal muscle thin filament motility in vitro. BiophysJ 72:1295-1307

Hoffmann B, Schmidt-Traub H, Perrot A, Osterziel KJ, Gessner R (2001) First mutation in cardiac troponin C, L29Q, in a patient with hypertrophic cardiomyopathy. Hum Mutat 17:524

Howarth JW, Meller J, Solaro RJ, Trewhella J, Rosevear PR (2007) Phosphorylation-dependent conformational transition of the cardiac specific $\mathrm{N}$-extension of troponin I in cardiac troponin. J Mol Biol 373:706-722. https://doi.org/10.1016/j.jmb.2007.08.035

Husic BE, Pande VS (2018) Markov state models: from an art to a science. J Am Chem Soc 140:2386-2396. https://doi.org/10.1021/ jacs.7b12191

Hwang PM, Cai F, Pineda-Sanabria SE, Corson DC, Sykes BD (2014) The cardiac-specific N-terminal region of troponin I positions the regulatory domain of troponin C. Proc Natl Acad Sci U S A 111:14412-14417. https://doi.org/10.1073/pnas.1410775111

Kaski JP, Burch M, Elliott PM (2007) Mutations in the cardiac Troponin $\mathrm{C}$ gene are a cause of idiopathic dilated cardiomyopathy in childhood. Cardiol Young 17:675-677

Kekenes-Huskey PM, Lindert S, McCammon JA (2012) Molecular basis of calcium-sensitizing and desensitizing mutations of the human cardiac troponin $\mathrm{C}$ regulatory domain: a multi-scale simulation study. PLoS Comput Biol 8:e1002777. https://doi. org/10.1371/journal.pcbi.1002777 
Kiani FA, Lehman W, Fischer S, Rynkiewicz MJ (2018) Spontaneous transitions of actin-bound tropomyosin toward blocked and closed states. J Gen Physiol. https://doi.org/10.1085/jgp.201812188

Knowles AC, Irving M, Sun Y-B (2012) Conformation of the troponin core complex in the thin filaments of skeletal muscle during relaxation and active contraction. J Mol Biol 421:125-137. https://doi. org/10.1016/j.jmb.2012.05.005

Kruger M et al (2005) Effects of the mutation R145G in human cardiac troponin I on the kinetics of the contraction-relaxation cycle in isolated cardiac myofibrils. J Physiol 564:347-357

Lang R, Gomes AV, Zhao J, Housmans PR, Miller T, Potter JD (2002) Functional analysis of a troponin I (R145G) mutation associated with familial hypertrophic cardiomyopathy. J Biol Chem 277:11670-11678

Lehman W (2016) Thin filament structure and the steric blocking model. Comprehensive physiology, vol 6. Wiley, Hoboken, pp 1043-1069. https://doi.org/10.1002/cphy.c150030

Lehman W (2017) Switching muscles on and off in steps: the McKillop-geeves three-state model of muscle regulation. Biophys J 112:2459-2466. https://doi.org/10.1016/j.bpj.2017.04.053

Li AY, Stevens CM, Liang B, Rayani K, Little S, Davis J, Tibbits GF (2013) Familial hypertrophic cardiomyopathy related cardiac troponin C L29Q mutation alters length-dependent activation and functional effects of phosphomimetic troponin I*. PLoS ONE 8:e79363. https://doi.org/10.1371/journal.pone.0079363

Lindert S, Cheng Y, Kekenes-Huskey P, Regnier M, McCammon JA (2015) Effects of HCM cTnI mutation R145G on troponin structure and modulation by PKA phosphorylation elucidated by molecular dynamics simulations. Biophys J 108:395-407. https ://doi.org/10.1016/j.bpj.2014.11.3461

Maier JA, Martinez C, Kasavajhala K, Wickstrom L, Hauser KE, Simmerling C (2015) ff14SB: improving the accuracy of protein side chain and backbone parameters from ff99SB. J Chem Theor Comp 11:3696-3713. https://doi.org/10.1021/acs.jctc.5b00255

Malnic B, Farah CS, Reinach FC (1998) Regulatory properties of the $\mathrm{NH} 2$ - and $\mathrm{COOH}$-terminal domains of troponin T, ATPase activation and binding to troponin I and troponin $\mathrm{C}$. J Biol Chem 273:10594-10601

Manning EP, Tardiff JC, Schwartz SD (2011) A model of calcium activation of the cardiac thin filament. Biochemistry 50:7405-7413. https://doi.org/10.1021/bi200506k

Manning EP, Tardiff JC, Schwartz SD (2012) Molecular effects of familial hypertrophic cardiomyopathy-related mutations in the TNT1 domain of cTnT. J Mol Biol 421:54-66. https://doi. org/10.1016/j.jmb.2012.05.008

Marston SB (2011) How do mutations in contractile proteins cause the primary familial cardiomyopathies? J Cardiovasc Transl Res 4:245-255. https://doi.org/10.1007/s12265-011-9266-2

Marston S (2016) Why is there a limit to the changes in myofilament $\mathrm{Ca}^{2+}$-sensitivity associated with myopathy causing mutations? Front Physiol. https://doi.org/10.3389/fphys.2016.00415

Marston S, Redwood CS (2003) Modulation of thin filament activation by breakdown or isoform switching of thin filament proteins physiological and pathological implications. Circ Res 93:1170-1178

Marston S et al (2013) Mutations in repeating structural motifs of tropomyosin cause gain of function in skeletal muscle myopathy patients. Hum Mol Genet 22:4978-4987. https://doi.org/10.1093/ $\mathrm{hmg} / \mathrm{ddt} 345$

Memo M et al (2013) Mutations in thin filament proteins that cause familial dilated cardiomyopathy uncouple troponin I phosphorylation from changes in myofibrillar $\mathrm{Ca}^{2+}$-sensitivity. Cardiovasc Res 99:65-73. https://doi.org/10.1093/cvr/cvt071

Messer A, Marston S (2014) Investigating the role of uncoupling of Troponin I phosphorylation from changes in myofibrillar $\mathrm{Ca}^{2+}$-sensitivity in the pathogenesis of cardiomyopathy. Front Physiol 5:315. https://doi.org/10.3389/fphys.2014.00315
Messer A et al (2016) Mutations in troponin T associated with Hypertrophic Cardiomyopathy increase $\mathrm{Ca}^{2+}$-sensitivity and suppress the modulation of $\mathrm{Ca}^{2+}$-sensitivity by troponin I phosphorylation. Arch Biochem Biophys 601:113-120

Messer AE, Chan J, Daley A, Copeland O, Marston SB, Connolly DJ (2017) Investigations into the sarcomeric protein and $\mathrm{Ca}^{2+}$-regulation abnormalities underlying hypertrophic cardiomyopathy in cats (Felix catus). Front Physiol 8:348. https://doi. org/10.3389/fphys.2017.00348

Metskas LA, Rhoades E (2015) Conformation and dynamics of the troponin I C-terminal domain: combining single-molecule and computational approaches for a disordered protein region. J Am Chem Soc 137:11962-11969. https://doi.org/10.1021/jacs.5b04471

Mittmann K, Jaquet K, Heilmeyer LM Jr (1990) A common motif of two adjacent phosphoserines in bovine, rabbit and human cardiac troponin I. FEBS Lett 273:41-45

Murakami K, Yumoto F, Ohki SY, Yasunaga T, Tanokura M, Wakabayashi $\mathrm{T}$ (2005) Structural basis for $\mathrm{Ca}^{2+}$-regulated muscle relaxation at interaction sites of troponin with actin and tropomyosin. J Mol Biol 352:178-201

Murakami K et al (2008) Structural basis for tropomyosin overlap in thin (actin) filaments and the generation of a molecular swivel by troponin-T. Proc Natl Acad Sci U S A 105:7200-7205. https://doi. org/10.1073/pnas.0801950105

Orzechowski M, Moore JR, Fischer S, Lehman W (2014) Tropomyosin movement on F-actin during muscle activation explained by energy landscapes. Arch Biochem Biophys 545C:63-68. https:// doi.org/10.1016/j.abb.2014.01.001

Orzechowski M, Lehman W, Cammarato A (2015) Docking troponin T onto tropomyosin. J Musc Res Cell Motil 36:541-542

Palm T, Graboski S, Hitchcock-DeGregori SE, Greenfield NJ (2001) Disease-causing mutations in cardiac troponin T: identification of a critical tropomyosin-binding region. Biophys J 81:2827-2837

Papadaki M, Marston SB (2016) The importance of intrinsically disordered segments of cardiac troponin in modulating function by phosphorylation and disease-causing mutations. Front Physiol 7:735. https://doi.org/10.3389/fphys.2016.00508

Paul DM, Squire JM, Morris EP (2017) Relaxed and active thin filament structures; a new structural basis for the regulatory mechanism. J Struct Biol 197:365-371. https://doi.org/10.1016/j.jsb.2017.01.004

Pirani A et al (2006) An atomic model of the thin filament in the relaxed and $\mathrm{Ca}^{2+}$-activated states. J mol Biol 357:707-717. https ://doi.org/10.1016/j.jmb.2005.12.050

Poole KJ et al (2006) A comparison of muscle thin filament models obtained from electron microscopy reconstructions and lowangle X-ray fibre diagrams from non-overlap muscle. J Struct Biol $155: 273-284$

Prinz J-H et al (2011) Markov models of molecular kinetics: generation and validation. J Chem Phys 134:174105. https://doi. org/10.1063/1.3565032

Robertson SP, Johnson JD, Holroyde MJ, Kranias EG, Potter JD, Solaro RJ (1982) The effect of troponin I phosphorylation on the $\mathrm{Ca}^{2+}$-binding properties of the $\mathrm{Ca}^{2+}$-regulatory site of bovine cardiac troponin. J Biol Chem 257:260-263

Robertson IM, Sevrieva I, Li MX, Irving M, Sun YB, Sykes BD (2015) The structural and functional effects of the familial hypertrophic cardiomyopathy-linked cardiac troponin C mutation, L29Q. J Mol Cell Cardiol 87:257-269. https://doi.org/10.1016/j.yjmcc .2015.08.017

Schaertl S, Lehrer SS, Geeves MA (1995) Separation and characterization of the two functional regions of troponin involved in muscle thin filament regulation. Biochemistry 34:15890-15894

Sevrieva I, Knowles AC, Kampourakis T, Sun Y-B (2014) Regulatory domain of troponin moves dynamically during activation of cardiac muscle. J Mol Cell Cardiol 75:181-187. https://doi. org/10.1016/j.yjmcc.2014.07.015 
Sheehan A et al (2018) Molecular defects in cardiac myofilament $\mathrm{Ca}^{2+}$-regulation due to cardiomyopathy-linked mutations can be reversed by small molecules binding to troponin. Front Physiol. https://doi.org/10.3389/fphys.2018.00243

Shoemaker BA, Portman JJ, Wolynes PG (2000) Speeding molecular recognition by using the folding funnel: the fly-casting mechanism. Proc Natl Acad Sci U S A 97:8868-8873. https://doi. org/10.1073/pnas.160259697

Solaro RJ, Kobayashi T (2011) Protein phosphorylation and signal transduction in cardiac thin filaments. J Biol Chem 286:99359940. https://doi.org/10.1074/jbc.R110.197731

Solaro RJ, Moir AGJ, Perry SV (1976) Phosphorylation of troponin I and the inotropic effect of adrenaline in the perfused rabbit heart. Nature 262:615-616

Spudich JA (2014) Hypertrophic and dilated cardiomyopathy: four decades of basic research on muscle lead to potential therapeutic approaches to these devastating genetic diseases. Biophys $\mathbf{J}$ 106:1236-1249. https://doi.org/10.1016/j.bpj.2014.02.011

Stevens CM, Rayani K, Singh G, Lotfalisalmasi B, Tieleman DP, Tibbits GF (2017) Changes in the dynamics of the cardiac troponin C molecule explain the effects of $\mathrm{Ca}^{2+}$-sensitizing mutations. J Biol Chem 292:11915-11926. https://doi.org/10.1074/jbc.m116.77077 6

Takeda N, Yamashita A, Maeda K, Maeda Y (2003) Structure of the core domain of human cardiac troponin in the $\mathrm{Ca}^{2+}$-saturated form. Nature 424:35-41

Thierfelder L et al (1994) Alpha-tropomyosin and cardiac troponin T mutations cause familial hypertrophic cardiomyopathy: a disease of the sarcomere. Cell 77:701-712. https://doi.org/10.1016/00928674(94)90054-X

Van Eyk JE et al (1997) Distinct regions of troponin I regulate $\mathrm{Ca} 2+$-dependant activation and $\mathrm{Ca}^{2+}$-sensitivity of the actoS1-TM ATPase activity of the thin filament. J Biol Chem 272:10529-10537

Vikhorev PG, Song W, Wilkinson R, Copeland O, Messer AE, Ferenczi MA, Marston SB (2014) The dilated cardiomyopathy-causing mutation ACTC E361G in cardiac muscle myofibrils specifically abolishes modulation of $\mathrm{Ca}(2+)$ regulation by phosphorylation of troponin I. Biophys J 107:2369-2380. https://doi.org/10.1016/j. bpj.2014.10.024

Vinogradova MV, Stone DB, Malanina GG, Karatzaferi C, Cooke R, Mendelson RA, Fletterick RJ (2005) $\mathrm{Ca}^{2+}$-regulated structural changes in troponin. Proc Natl Acad Sci U S A 102:5038-5045

Wilkinson R, Song W, Smoktunowicz N, Marston S (2015) A dilated cardiomyopathy mutation blunts adrenergic response and induces contractile dysfunction under chronic angiotensin II stress American journal of physiology Heart and circulatory physiology 309:H1936-H1946

Williams MR, Lehman SJ, Tardiff JC, Schwartz SD (2016) Atomic resolution probe for allostery in the regulatory thin filament. Proc Natl Acad Sci U S A. https://doi.org/10.1073/pnas.1519541113

Yang S, Barbu-Tudoran L, Orzechowski M, Craig R, Trinick J, White H, Lehman W (2014) Three-dimensional organization of troponin on cardiac muscle thin filaments in the relaxed state. Biophys $\mathbf{J}$ 106:855-864. https://doi.org/10.1016/j.bpj.2014.01.007

Zamora JE (2019) Investigating cardiomyopathies with atomistic simulations of cardiac Troponin, PhD Thesis. Imperial College London

Zamora JE, Papadaki M, Messer AE, Marston SB, Gould IR (2016) Troponin structure: its modulation by $\mathrm{Ca}(2+)$ and phosphorylation studied by molecular dynamics simulations. Phys Chem Chem Phys 18:20691-20707. https://doi.org/10.1039/c6cp02610a

Zhang XL, Tibbits GF, Paetzel M (2013) The structure of cardiac troponin $\mathrm{Cd}^{2+}$ regulatorydomain with bound $\mathrm{Cd}$ reveals a closedconformation and unique ion coordination. Acta Cryst 69:722-734. https://doi.org/10.1107/s0907444913001182

Publisher's Note Springer Nature remains neutral with regard to jurisdictional claims in published maps and institutional affiliations. 